\title{
Implications of a scalar dark force for terrestrial experiments
}

\author{
Sean M. Carroll, ${ }^{1, *}$ Sonny Mantry, ${ }^{2, \dagger}$ and Michael J. Ramsey-Musolf ${ }^{2,1, \ddagger}$ \\ ${ }^{1}$ California Institute of Technology, Pasadena, California 91125, USA \\ ${ }^{2}$ University of Wisconsin-Madison, Madison, Wisconsin 53706, USA
}

(Received 20 October 2009; published 5 March 2010)

\begin{abstract}
A long-range intergalactic force between dark matter (DM) particles, mediated by an ultralight scalar, is tightly constrained by galactic dynamics and large scale structure formation. We examine the implications of such a "dark force" for several terrestrial experiments, including Eötvös tests of the Weak Equivalence Principle (WEP), direct-detection DM searches, and collider studies. The presence of a dark force implies a nonvanishing effect in Eötvös tests that could be probed by current and future experiments depending on the DM model. For scalar DM that is a singlet under the standard model gauge groups, a dark force of astrophysically relevant magnitude is ruled out in large regions of parameter space by the DM relic density and WEP constraints. WEP tests also imply constraints on the Higgs-exchange contributions to the spinindependent (SI) DM-nucleus direct-detection cross section. For WIMP scenarios, these considerations constrain Higgs-exchange contributions to the SI cross section to be subleading compared to gauge-boson mediated contributions. In multicomponent DM scenarios, a dark force would preclude large shifts in the rate for Higgs decay to two photons associated with DM-multiplet loops that might otherwise lead to measurable deviations at the LHC or a future linear collider. The combination of observations from galactic dynamics, large scale structure formation, Eötvös experiments, DM-direct-detection experiments, and colliders can further constrain the size of new long-range forces in the dark sector.
\end{abstract}

DOI: 10.1103/PhysRevD.81.063507

PACS numbers: 95.35.+d, 98.80.Cq

\section{INTRODUCTION}

There is now compelling evidence for the $\Lambda \mathrm{CDM}$ model or the "standard model" of cosmology according to which the energy of the universe is about $74 \%$ dark energy, $22 \%$ dark matter (DM), and $4 \%$ baryonic matter. There have been independent confirmations of the dark energy component of the universe from observations of high redshift Type Ia supernovae [1-5]. The evidence for DM is even more compelling from the study of galactic rotation curves [6-8], acoustic oscillations in the cosmic microwave background [9-12], large scale structure formation [13,14], and gravitational lensing $[15,16]$. In spite of such strong evidence for the existence of dark energy and DM, almost nothing is known about their properties. The simplest explanation of dark energy is a small but nonzero cosmological constant. The DM properties such as its mass, quantum numbers, and interactions with the standard model (SM) remain unknown. Furthermore, it remains to be seen if there is only one type of DM particle responsible for all of the observational evidence, or if there exists a rich spectrum of DM particles analogous to the complexity seen in the visible sector. Many experiments are underway to detect DM and determine its properties. Ground based direct-detection experiments $[17,18]$ put limits on the DM mass and the strength of its interaction with baryonic matter from observations of recoiling nuclei. Experiments [19-22] studying cosmic rays from the galactic halo have

\footnotetext{
*seancarroll@gmail.com

mantry@wisc.edu

†jirm@physics.wisc.edu
}

recently seen indications of an electron/positron excess, which could be interpreted as evidence for DM annihilation, and can constrain the DM mass and interactions. There has also been a recent proposal to observe a possible DM magnetic moment via the gyromagnetic Faraday effect [23,24].

Another set of experiments are devoted to question of whether DM couples to new long-range forces of intergalactic range which could be observed as an apparent violation of the Weak Equivalence Principle (WEP) in the dark sector. There exist a variety of scenarios for new interactions confined solely to the dark sector and the possibility that they might be observed as an apparent WEP violation. The possibility of much shorter range gauge or Yukawa forces confined to the dark sector have been studied in other contexts [25-32] and also referred to as a "dark force". In this work however, we focus on a long-range intergalactic dark forces, mediated by an ultralight scalar, and study its implications for terrestrial experiments. For this scenario, the dark force can be communicated to ordinary matter via virtual DM loops that connect the ultralight scalar with ordinary matter, as long as the DM candidate is not sterile. This mechanism will give rise to effects in terrestrial experiments. We investigate the resulting impact on DM-detection experiments, laboratory based WEP tests, or even studies of Higgs boson properties at colliders. Constraints on an apparent WEP violation in ordinary matter induced dark forces were recently studied in [33,34]. In addition, a connection between direct DM-detection experiments and WEP tests was shown in [33]. 
Many models that contain the interaction of an ultralight scalar with DM [35-46] have been proposed to explain features in the DM distribution and explore the possibility of DM-quintessence interactions. More recently, work with nonuniversal scalar-tensor theories of gravity with the Abnormally Weighting Energy (AWE) Hypothesis [47,48] also invoke couplings of an ultralight scalar to the dark sector as a way of explaining the observed cosmic acceleration even in the absence of a dark energy fluid. Constraints on such scenarios from big bang nucleosynthesis have also been studied [49]. There are several other observational motivations, including higher than predicted supercluster densities [50] and voids [41,45] (for a summary see $[33,51])$. The existence of a long-range attractive Yukawa force between DM particles would accelerate structure formation and could help explain some of these observations. Strong constraints on such a dark force are derived from observations of DM dynamics in the tidal stream of the Sagittarius dwarf galaxy [51,52], which indicate a force with strength less that $20 \%$ of gravity for a range of about $20 \mathrm{kpc}$. However, new observational systematic errors have been recently discovered [53] that could require a revision of this result, perhaps allowing for a stronger dark force. A more recent analysis [54] considers the effect of a dark force on the evolution of density perturbations and the resulting impact on the CMB spectrum. This analysis constrains the strength of a dark force to be less than $5 \%$ of gravity.

From a purely theoretical perspective, the existence of an ultralight scalar $\phi$ with mass $m_{\phi}<10^{-25} \mathrm{eV}$, able to mediate a long-range force over scales of interest to galactic dynamics, would introduce a new hierarchy in addition to that between the weak scale $m_{W} \sim 100 \mathrm{GeV}$ and the Planck scale $M_{P} \sim 10^{19} \mathrm{GeV}$. However, as we still await experimental evidence for a mechanism to explain the hierarchy between the weak and Planck scales, and in light of the discovery of an unnaturally small cosmological constant, we keep an open mind and do not attempt to provide an explanation for the ultralight scalar mass. We assume the existence of a finely-tuned ultralight scalar mediating a long-range force dark force and study its consequences for terrestrial experiments.

In what follows, we amplify on our earlier work [34] and that of Ref. [33], using simple DM scenarios to illustrate the prospective implications of long-range scalar dark forces for terrestrial experiments. We study three representative minimal DM scenarios to explore the range of possible implications: scalar DM that is a singlet with respect to SM gauge interactions; scalar DM that is the neutral component of a real $\mathrm{SU}(2)_{L}$ triplet which is a triplet of $\mathrm{SU}(2)_{L}$ with zero hypercharge; and fermionic DM that lives in a vectorlike representation of $\mathrm{SU}(2)_{L}$ where the left and right handed components of the Dirac spinor transform in the same representation of $\mathrm{SU}(2)_{L}$. Our main conclusions are: (i) The presence of a dark force implies a nonzero effect in Eötvös experiments if the DM interacts with standard model (SM) fields. For scalar singlet DM, this effect arises from DM loop-induced mixing between the ultralight scalar and the SM Higgs generated from nonrenormalizable operators, while for representative WIMP scenarios (scalar or fermionic) additional contributions arise from DM loopinduced nonrenormalizable operators that couple the ultralight scalar directly to matter. We derive orderof-magnitude expectations for the minimum size of these effects for these representative scenarios as illustrated in Fig. 4 for WIMP DM and Table. II for scalar singlet DM. For a dark force with strength roughly $20 \%$ of gravity, one could expect a nonvanishing effect, for nonminimal WIMP DM models and in certain regions of parameter space of scalar singlet DM models, within reach of future approved Eötvös experiments such as Microscope [55] able to detect anomalous accelerations to a sensitivity of $\Delta a / a \sim 10^{-15}$. The MiniSTEP experiment [56] with an increased sensitivity of $\Delta a / a \sim 10^{-18}$, currently under study by NASA and the ESA, could see nonvanishing effects in minimal WIMP models which can induce effects starting with two-loop diagrams involving virtual DM.

(ii) For scalar singlet DM, a dark force of astrophysical relevance, is already ruled out in large regions of parameter space. The bounds from Eötvös experiments constrain the size of DM-Higgs interaction which determines the relic density, along with other known SM interactions, for a fixed value of the Higgs mass. In large regions of parameter space, the bound on DM-Higgs interactions implies a suppression in the DM annihilation rate resulting in a relic density that over-closes the universe. As a result, relic density considerations in scalar singlet DM models can yield the strongest bounds on the size of a dark force.

(iii) The constraints derived on the DM-Higgs interactions lead to upper bounds on the magnitude of Higgs-exchange contributions to DM-nucleus cross sections. These bounds depend on the Higgs mass, implying that a combination of direct-detection experiments and Higgs boson discovery could be used to test simple scenarios for dark forces. In particular, Higgs-exchange contributions dominate the SI scalar singlet DM-nucleus cross section, so that dark force considerations-together with the observed DM relic density-imply constraints on the entire cross section. In contrast, WIMP-nucleus cross sections receive contributions from electroweak gauge-boson-exchange that are not constrained by the presence of a dark force. As we show below, dark force considerations and present 
limits from Eötvös experiments imply that the Higgs-exchange contributions are subleading compared to those from gauge boson-exchange. The corresponding bounds for the scalar singlet and real triplet DM models are illustrated in Figs. 10. Tests of the WEP can only constrain the full DMnucleus cross section if the DM particles are singlets with respect to the SM gauge symmetries (see, e.g., [57-59] and references therein) so that elastic scattering proceeds only via Higgs exchange (at least at tree level).

(iv) In multicomponent WIMP DM scenarios, where one of the light $(\lesssim 200 \mathrm{GeV})$ DM components has a nonzero coupling to the Higgs, the presence of a dark force- together with tests of the WEPimply testable upper bounds on one-loop WIMPinduced shifts in the branching ratio for the SM Higgs to decay to two photons. These bounds generally lie well below the prospective sensitivities of LHC studies of $\operatorname{Br}(H \rightarrow \gamma \gamma)$ as seen in Fig. 12. The observation of a significant shift in this branching ratio would likely preclude this scenario for a dark force.

(v) The existence of an observable long-range dark force which requires $m_{\phi}<10^{-25} \mathrm{eV}$, implies restrictions in the space of finite renormalized parameters in addition to the usual fine-tuning of radiative corrections that are sensitive to the cutoff. We discuss these regions in parameter space and their implications for the observation of a dark force.

In arriving at these conclusions, we emphasize we have drawn upon representative cases rather than carrying out a comprehensive study. We expect that our conclusions will generalize to other DM scenarios, but do not preclude the possibility of exceptions in some cases. We also note that our analysis and conclusions differ from those of Ref. [33], who first observed that bounds on WEP and the presence of an astrophysically relevant dark force could imply constraints on DM-nucleus cross sections. The bounds obtained in that work lie well below the reach of future direct-detection experiments. In what follows, we argue that an effective operator analysis consistent with the finetuning needed to maintain a vanishingly small scalar mass implies considerably weaker bounds than given in Ref. [33].

The outline of the paper is as follows. In Sec. II we review the phenomenology of experimental WEP tests and establish notation. In Sec. III we review the derivation of the ultralight scalar coupling to macroscopic objects in terms of its couplings to the SM particles. In Sec. IV we discuss in a model independent way the mechanisms by which the ultralight scalar can couple to the SM. In Secs. V and VI we examine the experimental consequences of a dark force for various minimal DM models. In Sec. VII we discuss the regions in parameter space where an observable dark force is possible and how they relate to our analysis. We conclude in Sec. VIII.

\section{FIFTH-FORCE PHENOMENOLOGY}

We begin by considering the force between two bodies mediated by a scalar field $\phi$ with mass $m_{\phi}$. In the nonrelativistic limit, the Yukawa potential between a test body $i$ and a source $s$ separated by a distance $r$ is given (in units where $\hbar=c=1$ ) by

$$
V_{\phi}=-\xi_{i} \xi_{s} \frac{Q_{i} Q_{s}}{4 \pi r} e^{-m_{\phi} r},
$$

where $Q_{i, s}$ denote the charges of the test and source objects under the force mediated by $\phi$. The parameters $\xi_{i, s}$ are $^{1}$

$$
\xi_{i, s}= \begin{cases}1 & \text { for fermionic objects, } \\ \frac{1}{2 m_{i, s}} & \text { for scalar objects. }\end{cases}
$$

Note that the charges $Q_{i, s}$ are of mass dimension one and zero for scalar and fermionic objects, respectively, so that the equation is dimensionally consistent. These mass dimensions will become apparent when we study specific models. The Newtonian gravitational potential between a body with mass $M_{i}$ and a source with mass $M_{s}$ is

$$
V_{G}=-\frac{G M_{i} M_{s}}{r},
$$

where $G$ is Newton's constant. It is therefore convenient to write the total potential as

$$
V=-\frac{G M_{i} M_{s}}{r}\left(1+\alpha_{i s} e^{-m_{\phi} r}\right)
$$

where

$$
\alpha_{i s}=\frac{1}{4 \pi G} \frac{q_{i} q_{s}}{\mu_{i} \mu_{s}} \hat{\xi}_{i} \hat{\xi}_{s},
$$

is a dimensionless parameter characterizing the strength of the new force relative to gravity, expressed in terms of the charge-to-mass ratio $q / \mu=Q / M$, where $\mu$ is the mass in atomic mass units. The parameters $\hat{\xi}_{i, s}$ are

$$
\hat{\xi}_{i, s}= \begin{cases}1 & \text { for fermionic objects, } \\ \frac{1}{2 \mu_{i, s}} & \text { for scalar objects. }\end{cases}
$$

The parameter $\alpha_{i s}$ is not universal and in general depend on the composition of the macroscopic bodies acting as sources for $\phi$.

\footnotetext{
${ }^{1}$ The t-channel $\phi$ exchange amplitude is accompanied by an extra factor of $2 m_{i, s}$ for fermions relative to scalars. This is due to the fermionic spinor normalization $\bar{u}_{i, s} u_{i, s}=2 m_{i, s}$ in the nonrelativistic limit. These factors are absorbed by switching to states with normalization $\langle\mathbf{p} \mid \mathbf{q}\rangle=(2 \pi)^{3} \delta^{(3)}(\mathbf{p}-\mathbf{q})$ in order to compare with the nonrelativistic Born amplitude. For scalars we are then left with an additional factor of $\frac{1}{2 m_{i s}}$ in the potential relative to fermions.
} 
Eötvös experiments look for apparent violations of the equivalence principle by measuring the difference in acceleration of two test bodies of different compositions in the presence of a common source. Experimental constraints on new long-range composition-dependent forces are typically expressed in terms of the Eötvös parameter,

$$
\eta=2 \frac{\left|a_{1}-a_{2}\right|}{\left|a_{1}+a_{2}\right|} \simeq\left|\frac{\Delta a}{a}\right|,
$$

where $a_{i}$ is the total acceleration of object $i=1,2, \Delta a \equiv$ $a_{1}-a_{2}$, and $a$ is the universal gravitational acceleration in the absence of any new long-range forces. The last approximation made above is valid when the fifth force is weaker than gravity. From (4), the acceleration of object $i$ due to the source $s$ is

$$
a_{i}=\frac{\mathrm{GM}_{s}}{r^{2}}\left[1+\alpha_{i s}\left(1+m_{\phi} r\right) e^{-m_{\phi} r}\right] .
$$

The dark forces are constrained to be weaker than gravity, and we are interested in distances less than the Compton wavelength of the scalar, $r \ll m_{\phi}^{-1}$. The Eötvös parameter is then

$$
\eta_{s}^{1,2}=\frac{1}{4 \pi G}\left|\frac{q_{1} \hat{\xi}_{1}}{\mu_{1}}-\frac{q_{2} \hat{\xi}_{2}}{\mu_{2}}\right|\left|\frac{q_{s} \hat{\xi}_{s}}{\mu_{s}}\right| .
$$

Currently, the strongest limits on violations of the weak equivalence come from torsion balance Eötvös experiments [60] which give the constraints

$$
\begin{aligned}
& \eta_{E}^{\mathrm{Be}, \mathrm{Ti}}<(0.3 \pm 1.8) \times 10^{-13}, \\
& \eta_{\mathrm{DM}}^{\mathrm{Be}, \mathrm{Ti}}<(4 \pm 7) \times 10^{-5} .
\end{aligned}
$$

The Eötvös parameters $\eta_{E}^{\mathrm{Be}, \mathrm{Ti}}$ and $\eta_{\mathrm{DM}}^{\mathrm{Be}, \mathrm{Ti}}$ measure differential acceleration of laboratory test samples of Beryllium and Titanium with the Earth and galactic dark matter as the source bodies, respectively.

Future experiments, currently being studied, are expected to further improve the bound on the Eötvös parameter by several orders of magnitude as shown in Table I. The MiniSTEP experiment [56], currently under study, would use test objects of different composition orbiting earth in free fall and new technology to reduce thermal noise. If approved, this experiment is expected to achieve the highest sensitivity of $\eta \sim 10^{-18}$. The Microscope experiment,

TABLE I. Expected sensitivities for the Eötvös parameter in future experiments testing the WEP. The MiniSTEP experiment is currently under study by NASA and the ESA. Microscope has been approved and the Apollo (LLR) experiment is underway.

\begin{tabular}{lc}
\hline \hline Experiment & Expected Future Sensitivity in $\eta$ \\
\hline MiniSTEP [56] & $10^{-18}$ \\
Microscope [55] & $10^{-15}$ \\
Apollo (LLR) [61] & $10^{-14}$ \\
\hline \hline
\end{tabular}

which has been approved, uses the same principle but is expected to reach a sensitivity of $\eta \sim 10^{-15}$. In the method of Lunar Laser Ranging (LLR) used by the APOLLO collaboration [61], the differential acceleration of the Earth and Moon is measured in the presence of a source like the Sun or galactic dark matter. The APOLLO collaboration, which is currently underway, is expected to achieve a sensitivity of $\eta \sim 10^{-14}$ improving the current sensitivity for $\eta$ by an order-of-magnitude. Methods using atom interferometry [62] could reach a sensitivity of $\eta \sim$ $10^{-17}$.

These experiments are also sensitive to dark forces, if the DM has interactions with the SM. Through quantum effects involving virtual DM, dark forces will be communicated $[33,34]$ to ordinary matter and these effects can be tested in Eötvös experiments. A dark force is already constrained from an analysis of the tidal disruption in satellite galaxies [51]. This study constrains the coupling of $\phi$ to DM particles by putting bounds on the parameter $\beta$

$$
\beta=\frac{M_{P}}{\sqrt{4 \pi}} \frac{\left|g_{\chi}\right|}{M_{\chi}} \xi_{\chi}
$$

where we reserve the symbol $g_{\chi}$ for the DM charge under the fifth force, $M_{\chi}$ denotes the DM mass, $M_{P}=1 / \sqrt{G}$ is the Planck mass, and $\xi_{\chi}$ is as defined in Eq. (2). The coupling $g_{\chi}$ appears in the Lagrangian via interaction terms for fermionic ${ }^{2}$ and scalar DM of the form

$$
\delta \mathcal{L}= \begin{cases}g_{\chi} \bar{\chi} \chi \phi, & \text { fermionic DM } \\ g_{\chi} \chi^{\dagger} \chi \phi & \text { scalar DM }\end{cases}
$$

Thus, we see that for fermionic DM, $g_{\chi}$ is dimensionless and for scalar DM it has dimension one. From the analysis of tidal streams in the Sagittarius galaxy, Kamionkowski and Kesden [51] obtained the approximate upper bound of

$$
\beta \leqq 0.2 .
$$

Newly discovered systematic errors [53] could lead to a revision of this bound and more recently, the work of [63] showed the possibility of $\beta \sim 1$ consistent with observations of galactic dynamics. A more recent analysis [54] of the $\mathrm{CMB}$ and large scale structure formation gives a tighter bound of $\beta<0.05$. In this paper we use $\beta=0.2$ as a reference value for most discussions, and our results be straightforwardly translated to other values of $\beta$.

\section{LIGHT SCALAR COUPLING TO MACROSCOPIC OBJECTS}

The charge-to-mass ratio under a fifth force for an elementary particle is straightforward to obtain in terms

\footnotetext{
${ }^{2}$ For simplicity we assume that the fermionic DM is in a vectorlike gauge representation so that $\bar{\chi} \chi \phi$ is gauge invariant. For chiral DM, the coupling to $\phi$ can arise from higherdimension operators.
} 
of the Lagrangian parameters. For example, the charge-tomass ratio for elementary fermionic or scalar DM $\chi$ is given by

$$
\left(\frac{q}{\mu}\right)_{\chi}=\frac{g_{\chi}}{M_{\chi}} .
$$

This charge-to-mass ratio is obtained by computing the tree-level $\phi$ exchange diagram between two DM particles and taking the nonrelativistic limit to compare with Eq. (4).

For composite materials the calculation of the charge-tomass ratio is more complicated [64-67], as one has to take into account hadronic, nuclear, and atomic matrix elements of various operators containing SM fields that couple to $\phi$ as well as the effects of binding energy. In particular, one needs the charge-to-mass ratio for the various types of atoms that make up the laboratory test materials. We compute these ratios using an effective field theory valid near the nucleon mass scale that involves the light quarks $q=\{u, d, s\}$, gluons, the charged leptons $\ell=\{e, \mu\}$, the photon, and the light scalar $\phi$. All other heavier degrees of freedom have been integrated out. The interaction terms in this effective Lagrangian take the form:

$$
\begin{aligned}
\mathcal{L}_{\phi}= & \sum_{q} \frac{g_{q}}{m_{p}} m_{q} \bar{q} q \phi+\sum_{\ell} \frac{g_{\ell}}{m_{p}} m_{\ell} \bar{\ell} \ell \phi+c_{g} \phi G_{\mu \nu}^{a} G_{a}^{\mu \nu} \\
& +c_{\gamma} \phi F_{\mu \nu} F^{\mu \nu} .
\end{aligned}
$$

As we discuss below, the effects of the $\phi$ coupling to heavy quarks, the tau lepton, massive gauge bosons, and $\chi$ that have been integrated out are encoded in the operator coefficients $g_{q, \ell}$ and $c_{g, \gamma}$. We assume that the couplings of $\phi$ to the SM fermions are linearly proportional to the fermion mass. This will make the analysis simpler, as we will see, by allowing us to exploit the scale invariance of the energymomentum tensor. This assumption is realized in several types of DM models. The couplings $c_{g}$ and $c_{\gamma}$ can be straightforwardly computed in any given model. To illustrate, consider a model in which $\phi$ couples to the SM fermions at the electroweak scale as

$$
\begin{aligned}
\mathcal{L}_{\phi f \bar{f}}= & \sum_{q} \frac{g_{q}}{m_{p}} m_{q} \bar{q} q \phi+\sum_{\ell} \frac{g_{\ell}}{m_{p}} m_{\ell} \bar{\ell} \ell \phi+\frac{g_{\tau}}{m_{p}} m_{\tau} \bar{\tau} \tau \phi \\
& +\sum_{Q} \frac{g_{Q}}{m_{p}} m_{Q} \bar{Q} Q \phi,
\end{aligned}
$$

where the sum over $Q$ denotes a sum over the heavy $b, c, t$ quarks and all the couplings $g_{\ell, \tau, q, Q}$ above are independent of the SM fermion masses. One can then integrate out the heavy quarks and the tau lepton to obtain [64] the renormalization group invariant relations

$$
\begin{aligned}
m_{Q} \bar{Q} Q & =-\frac{\alpha_{s}}{12 \pi} G_{\mu \nu}^{a} G_{a}^{\mu \nu}-\frac{\alpha}{16 \pi} F_{\mu \nu} F^{\mu \nu}, \\
m_{\tau} \bar{\tau} \tau & =-\frac{\alpha}{16 \pi} F_{\mu \nu} F^{\mu \nu}
\end{aligned}
$$

to leading order in the heavy quark and tau lepton mass expansion and perturbation theory. Note that the right-hand side (rhs) above is independent of the heavy quark and tau lepton mass. In this case the couplings $c_{g}$ and $c_{\gamma}$ in Eq. (15) are given by

$$
\begin{aligned}
c_{g} & =-\frac{1}{m_{p}}\left(\sum_{Q} g_{Q}\right) \frac{\alpha_{s}}{12 \pi}, \\
c_{\gamma} & =-\frac{1}{m_{p}}\left(\sum_{Q} g_{Q}+g_{\tau}\right) \frac{\alpha}{48 \pi},
\end{aligned}
$$

at leading order. The mass operators on the left-hand side (lhs) of the equations in Eq. (17) appear in the QCD + QED energy-momentum tensor and are scale invariant, allowing us to evaluate $\alpha_{s}$ and $\alpha$ in Eq. (18) at the lowenergy scale of the effective theory (when taking the atomic matrix element). Because they do not run below the electroweak scale, couplings $g_{Q, \tau}$ are evaluated at that scale.

We now evaluate the coupling of $\phi$ to an atom [66-69] of type "A". Doing this allows us to determine the chargeto-mass ratio $q_{A} \hat{\xi}_{A} / \mu_{A}$ needed for Eötvös parameters, as seen from Eq. (9), if the test or source bodies are made up of atoms of type "A". We define the effective atomic coupling as

$$
\mathcal{L}_{A A \phi}= \begin{cases}g_{A} \bar{A} A \phi, & \text { fermionic atoms, } \\ g_{A} A^{\dagger} A \phi, & \text { scalar atoms }\end{cases}
$$

where the $A$ is the field that destroys the atomic state and again $g_{A}$ is dimensionless for a spin $1 / 2$ atom and has dimension one for a spin zero atom. While a similar approach can be applied to higher spin atoms we restrict our analysis to spin $1 / 2$ and 0 for the sake brevity and use these cases as illustrative examples rather than be exhaustive and cover all possibilities. We determine $g_{A}$ by a matching calculation

$$
\left\langle A\left|\mathcal{L}_{A A \phi}\right| A \phi\right\rangle=g_{A} \xi_{A}=\left\langle A\left|\mathcal{L}_{\phi}\right| A \phi\right\rangle,
$$

where we have used a nonrelativistic normalization for the atomic states $\langle A(p) \mid A(q)\rangle=(2 \pi)^{3} \delta^{3}(\vec{p}-\vec{q}), \quad \xi_{A}$ is the normalization factor defined in Eq. (2), and $\mathcal{L}_{\phi}$ is defined in (15). From Eq. (20), as explained in Appendix A, the general expression for the charge-to-mass ratio $q_{A} \hat{\xi}_{A} / \mu_{A}$ is

$$
\begin{aligned}
\hat{\xi}_{A}\left(\frac{q}{\mu}\right)_{A}= & \frac{g_{A} \xi_{A}}{M_{A}} \\
= & \frac{2 c_{g} g_{3}}{\beta_{3}}+\frac{1}{M_{A}}\left[Z\left(\zeta_{e} m_{e}+\sum_{q} \zeta_{q} m_{q} x_{q, p}\right)\right. \\
& \left.+(A-Z) \sum_{q} \zeta_{q} m_{q} x_{q, n}+\omega_{A}\right]
\end{aligned}
$$

where the quantity $\omega_{A}$ is given by 


$$
\omega_{A} \equiv \kappa\left\langle A\left|F^{\mu \nu} F_{\mu \nu}\right| A\right\rangle-\sum_{k} \zeta_{k} m_{k} \frac{d \mathcal{E}_{A}}{d m_{k}},
$$

$\mathcal{E}_{A}$ is the atomic binding energy as defined in Eq. (A5), the quantities $\zeta_{k}$ and $\kappa$ are given by

$$
\zeta_{k}=\frac{g_{k}}{m_{p}}-\frac{2 g_{3}}{\beta_{3}} c_{g}, \quad \kappa=c_{\gamma}-\frac{g_{3} \beta_{e}}{e \beta_{3}} c_{g},
$$

as in Eq. (A10), the $m_{e}$ term is the contribution from electrons in the atomic shells, and $x_{q, p}$ and $x_{q, n}$ denote the nucleon matrix elements

$$
x_{q, p}=\langle p|\bar{q} q| p\rangle, \quad x_{q, n}=\langle n|\bar{q} q| n\rangle,
$$

which have been measured [67,70], although large uncertainties remain. However, for our order-of-magnitude analysis we use the central values quoted in [70] which are listed in Eq. (A13) of appendix A. In Eq. (23), $\beta_{3}$ and $\beta_{e}$ denote the QCD and QED beta functions, respectively.

Using Eq. (21) in Eq. (9) for test objects made up of atoms with atomic weights $A_{1}$ and $A_{2}$, the general expression for the Eötvös parameter $\eta_{S}$ with source $S$ is

$$
\begin{aligned}
\eta_{S}= & \frac{M_{P}^{2}}{4 \pi}\left|\hat{\xi}_{S}\left(\frac{q}{\mu}\right)_{S}\right| \mid\left(\frac{Z_{1}}{M_{A_{1}}}-\frac{Z_{2}}{M_{A_{2}}}\right) \\
& \times\left(\zeta_{e} m_{e}+\sum_{q} \zeta_{q} m_{q} x_{q, p}\right)+\left(\frac{A_{1}-Z_{1}}{M_{A_{1}}}-\frac{A_{2}-Z_{2}}{M_{A_{2}}}\right) \\
& \times \sum_{q} \zeta_{q} m_{q} x_{q, n}+\left(\frac{\omega_{A_{1}}}{M_{A_{1}}}-\frac{\omega_{A_{2}}}{M_{A_{2}}}\right) \mid
\end{aligned}
$$

where $\left(\frac{q}{\mu}\right)_{S}$ denotes the charge-to-mass ratio for the source object and $A_{k}, Z_{k}(k=1,2)$ refer to the atomic weights and atomic numbers of the two laboratory samples. For orderof-magnitude estimates, we follow Ref. [66] and ignore binding energy effects, encoded in the quantities $\omega_{A_{1,2}}$. Setting $M_{A} \simeq A m_{N}$ for the atomic masses, we then obtain the simpler expression

$$
\begin{aligned}
\eta_{S} \simeq & \frac{M_{P}^{2}}{4 \pi m_{N}}\left|\hat{\xi}_{S}\left(\frac{q}{\mu}\right)_{S}\right| \\
& \times\left|\left(\frac{Z_{1}}{A_{1}}-\frac{Z_{2}}{A_{2}}\right)\left\{\zeta_{e} m_{e}+\sum_{q} \zeta_{q} m_{q}\left(x_{q, p}-x_{q, n}\right)\right\}\right| .
\end{aligned}
$$

From Eqs. (18) and (23), the parameters $\zeta_{k}$ appearing above are given by

$$
\zeta_{k}=\frac{1}{m_{p}}\left[g_{k}-\frac{2}{27} \sum_{Q} g_{Q}\right]
$$

at leading order. Here $g_{k}$ denotes the couplings of $\phi$ to the light quarks and charged leptons and $g_{Q}$ denotes its coupling to the heavy $(b, c, t)$ quarks. A special case that will be of particular interest in subsequent discussion occurs when the couplings to fermions are universal, apart from the fermion Yukawa couplings explicitly factored out via the factors of $m_{f}$ in Eqs. (15) and (16). Setting

$$
g_{k}=g_{Q} \equiv \bar{g}
$$

and $m_{p}=m_{n}=m_{N}$ leads to

$$
\begin{aligned}
\eta_{S}^{\text {univ }} \simeq & \bar{g}\left(\frac{M_{P}^{2}}{4 \pi m_{N}^{2}}\right)\left(\frac{7}{9}\right)\left|\hat{\xi}_{S}\left(\frac{q}{\mu}\right)_{S}\right| \\
& \times\left|\left(\frac{Z_{1}}{A_{1}}-\frac{Z_{2}}{A_{2}}\right)\left\{m_{e}+\sum_{q} m_{q}\left(x_{q, p}-x_{q, n}\right)\right\}\right| .
\end{aligned}
$$

Typical source objects ' $S$ ' used in Eötvös experiments include the Earth, the Sun, and galactic DM, and one needs to obtain their charge-to-mass ratio $\hat{\xi}_{S}\left(\frac{q}{\mu}\right)_{S}$ that appears in Eq. (29). If galactic DM is made of elementary particles, then as already discussed, the charge-to-mass ratio under the dark force is given by

$$
\left.\hat{\xi}_{S}\left(\frac{q}{\mu}\right)_{S}\right|_{S=\mathrm{DM}}=\left(\frac{g_{\chi}}{M_{\chi}}\right) \hat{\xi}_{\chi} .
$$

For objects like the Earth that are made up of many different types of atoms, the effective charge-to-mass ratio is obtained by a superposition of the couplings of $\phi$ to all the different atoms present in the object. In contrast to the situation for differences in charge-to-mass ratios for test bodies, it suffices to approximate this ratio for the bulk source object by ignoring atomic binding energy effects and summing over the couplings of $\phi$ to all the neutrons, protons, and electrons present. Doing so in the case of the Earth leads to

$$
\hat{\xi}_{E}\left(\frac{q}{\mu}\right)_{E} \simeq \frac{g_{p} N_{p}+g_{n} N_{n}+g_{e}\left(m_{e} / m_{N}\right) N_{e}}{m_{N}\left(N_{p}+N_{n}\right)+m_{e} N_{e}},
$$

where $N_{p}, N_{n}, N_{e}$ denote the total number of protons, neutrons, and electrons, respectively, that make up Earth and $g_{p}$ and $g_{n}$ denote the couplings of $\phi$ to protons and neutrons, respectively:

$$
g_{N}=\left\langle N\left|\mathcal{L}_{\phi f \bar{f}}\right| N\right\rangle,
$$

for $N=p$ or $n$. In the limit of a universal coupling as in Eq. (28), one can express $g_{N}$ in terms of $g_{h}$, the coupling of the Higgs to the nucleon worked out in [71,72], as

$$
g_{N}=g_{h} \bar{g}\left(\frac{v}{m_{N}}\right)
$$

where $v=246 \mathrm{GeV}$ is the vacuum expectation value of the neutral component of the Higgs doublet and the expression for $g_{h}$ is [71,72]

$$
g_{h}=\left\langle N\left|\left(\sum_{q} \frac{m_{q}}{v} \bar{q} q+\sum_{Q} \frac{m_{Q}}{v} \bar{Q} Q\right)\right| N\right\rangle .
$$

Using similar methods to those employed to determine $g_{A}$ and ignoring small difference between the neutron and 
proton coupling, one has [71-73]

$$
g_{h} \simeq 1.71 \times 10^{-3} .
$$

The resulting expression for the Earth's charge-to-mass ratio in this case is

$$
\begin{aligned}
&\left.\hat{\xi}_{E}\left(\frac{q}{\mu}\right)_{E}\right|_{\text {univ }} \simeq \bar{g}\left(\frac{v}{m_{N}^{2}}\right) \frac{g_{h}\left(N_{p}+N_{n}\right)+\left(m_{e} / v\right) N_{e}}{\left(N_{p}+N_{n}\right)+\left(m_{e} / m_{N}\right) N_{e}} \\
& \simeq 0.0017 \bar{g}\left(\frac{v}{m_{N}^{2}}\right) .
\end{aligned}
$$

The number of protons, neutrons, and electrons are $N_{p} \simeq$ $1.9 \times 10^{51}, N_{n} \simeq 2.0 \times 10^{51}$, and $N_{e} \simeq 1.9 \times 10^{51}$ respectively. We will make use of Eq. (36) in what follows.

\section{LIGHT SCALAR COUPLING TO THE STANDARD MODEL}

We now give a general discussion of the ways in which an ultralight singlet scalar that mediates the dark force can couple to SM fields. In doing so, we will lay the groundwork for calculating the parameters $g_{f}(f=q, Q, \ell), c_{g}$, and $c_{\gamma}$ of Eq. (15) and (16) or equivalently the parameters $\zeta_{k}$ and $\kappa$ in Eqs. (23) and (27). In general a singlet scalar can couple to SM fermions and gauge bosons only via nonrenormalizable operators. ${ }^{3}$ However, it can couple to the Higgs sector via both renormalizable and nonrenormalizable interactions. We discuss these mechanisms for the singlet scalar to couple to ordinary matter in this section. We also address the need for fine-tuning of the ultralight scalar mass when its interactions with the SM are nonnegligible, looking ahead to a similar issue when we consider its coupling to DM.

\section{A. Coupling to the Higgs sector}

We assume that the mediator of the dark force carries no SM charges and that it can be described by a gauge singlet $S$. There exist no renormalizable couplings of such a singlet scalar to the SM fermions or gauge bosons, but it can couple to the SM Higgs doublet with operators of mass dimension $n \leq 4$. After electroweak symmetry breaking, the $n=3$ interaction $H^{\dagger} H S$ will generate mixing between $S$ and the neutral component of the Higgs doublet, $h$. We will identify the ultralight force-carrying scalar $\phi$ with the lighter mass eigenstate, and the heavier eigenstate with the physical Higgs boson. The Lagrangian for the singlet $S$ including its renormalizable and super-renormalizable interactions is given by

$$
\mathcal{L}=\frac{1}{2} \partial_{\mu} S \partial^{\mu} S-V(H, S)
$$

\footnotetext{
${ }^{3}$ We do not consider right handed neutrino fields which could couple to the singlet scalar at the renormalizable level. Including such interactions will not affect our analysis and we thus ignore them for simplicity and brevity.
}

where the potential is ${ }^{4}$

$$
\begin{aligned}
V(H, S)= & \frac{\lambda}{4}\left(H^{\dagger} H-\frac{v^{2}}{2}\right)^{2}+\frac{\delta_{1}}{2}\left(H^{\dagger} H-\frac{v^{2}}{2}\right) S \\
& +\frac{\kappa_{2}}{2} S^{2}+\frac{\kappa_{3}}{3} S^{3}+\frac{\kappa_{4}}{4} S^{4} .
\end{aligned}
$$

We have shifted the scalar $S$ so that it has no tree-level vacuum expectation value. We follow the notation of Refs. [57,74], which explored the presence of such a singlet scalar in the context of collider phenomenology. The parameters $\delta_{1,2}$ may arise from a more fundamental theory of which the $S$ is a residual, low-energy degree of freedom. As we discuss below, they may also receive contributions from DM loops if the DM particles couple to the both $H$ and $S$.

After electroweak symmetry breaking the $H^{\dagger} H S$ interaction induces mixing between the Higgs boson $h$ and the scalar $S$. In unitary gauge the neutral component of the Higgs doublet $H$ is given by

$$
H^{0}=\frac{v+h}{\sqrt{2}}
$$

and the mass terms in the potential are

$$
V_{\text {mass }}=\frac{1}{2}\left(\mu_{h}^{2} h^{2}+\mu_{S}^{2} S^{2}+\mu_{h S}^{2} h S\right),
$$

where

$$
\mu_{h}^{2}=\frac{\lambda v^{2}}{2}, \quad \mu_{S}^{2}=\kappa_{2}+\frac{\delta_{2} v^{2}}{2}, \quad \mu_{h S}^{2}=\delta_{1} v .
$$

The mass eigenstates $h_{ \pm}$in terms of $S$ and $h$ can be written in terms of a mixing angle $\theta$ as

$$
\begin{gathered}
h_{-}=S \cos \theta-h \sin \theta, \quad h_{+}=S \sin \theta+h \cos \theta, \\
\tan \theta=\frac{x}{1+\sqrt{1+x^{2}}},
\end{gathered}
$$

with corresponding masses

$$
m_{ \pm}^{2}=\frac{\mu_{h}^{2}+\mu_{S}^{2}}{2} \pm \frac{\mu_{h}^{2}-\mu_{S}^{2}}{2} \sqrt{1+x^{2}},
$$

and we have defined

$$
x \equiv \frac{\mu_{h S}^{2}}{\mu_{h}^{2}-\mu_{S}^{2}} .
$$

We assume that $m_{-}^{2} \ll m_{+}^{2}$, so that the physical Higgs boson and light scalar are $h_{+}$and $h_{-}$respectively. The light scalar $h_{-}$can couple to quarks and charged leptons through its mixing with the Higgs as shown in Eq. (42). We identify

\footnotetext{
${ }^{4}$ Here we also assume that $\delta_{1}$ or $\kappa_{3}$ are nonzero so that the potential has no discrete symmetry. In this case, the existence of a vacuum expectation value for $S$ (before the linear shift) would not lead to potentially problematic cosmological domain walls.
} 


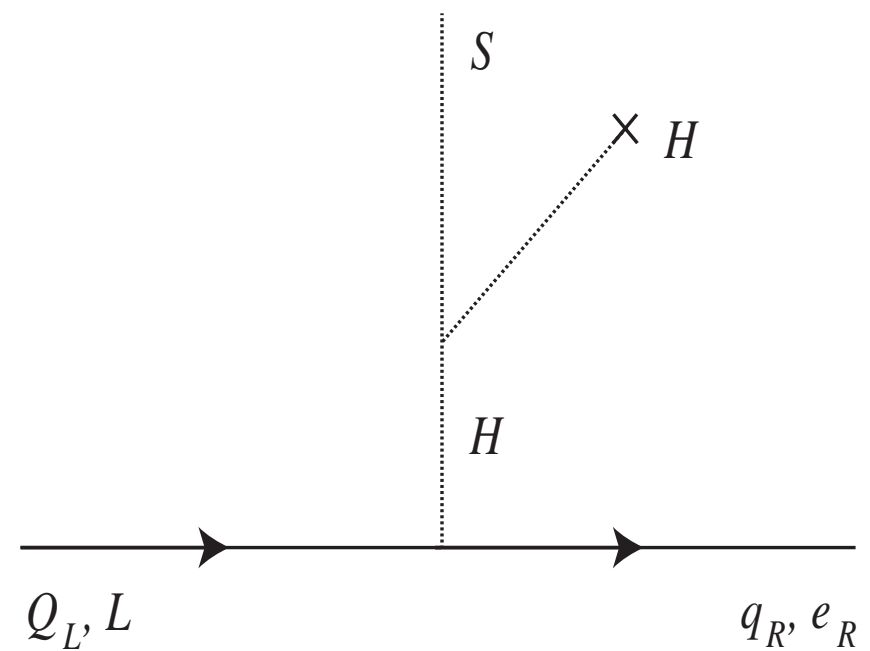

FIG. 1. Interaction of $S$ with SM fermions by mixing with the Higgs via the operator $S H^{\dagger} H$. Here, " $X$ " denotes the vacuum expectation value of the Higgs doublet.

the light scalar that mediates the long-range force as

$$
\phi \equiv h_{-}, \quad m_{\phi}=m_{-} .
$$

The scalar $\phi$ couples to the SM fermions through its Higgs component, giving rise to the couplings $g_{f}$, where $f$ denotes any of the light quarks $q=u, d, s$, charged leptons $\ell=e, \mu, \tau$, or heavy quarks $Q=c, b, t$. One has

$$
g_{f}=-\sin \theta \frac{m_{p}}{m_{f}} \frac{m_{f}}{v}=-\sin \theta \frac{m_{p}}{v}
$$

where the extra factor of $m_{p} / m_{f}$ after the first equality is included to be consistent with the convention in Eq. (15). This process is depicted in Fig. 1. We see that in this mechanism the coupling of $\phi$ to ordinary matter is proportional to $\sin \theta$, with the constant of proportionality given entirely in terms of known quantities. The mixing angle $\theta$ will also receive corrections at the loop level and in the rest of the analysis we assume that $\theta$ is the renormalized mixing angle.

For later use, we note that in the limit that $\mu_{h} \simeq m_{h} \gg$ $\mu_{S}$ corresponding to a small mixing angle $\theta$, we can write

$$
m_{\phi}^{2} \simeq \mu_{S}^{2}-\frac{\mu_{h S}^{4}}{4 m_{h}^{2}} .
$$

The existence of an ultralight scalar that can mediate a dark force over intergalactic distances requires $m_{\phi}<10^{-25} \mathrm{eV}$. In addition to the usual fine-tuning of the parameters $\mu_{S}$ and $\mu_{h S}$ against radiative corrections sensitive to the cutoff (see Sec. IV C), the finite renormalized parameters $\mu_{S}$ and $\mu_{h S}$ are restricted in parameter space to satisfy the condition $m_{\phi}<10^{-25} \mathrm{eV}$ in Eq. (47). As we will discuss in Sec. VII in more detail, this gives rise to three types of regions in parameter space. In the first region, $\mu_{S}$ and $\mu_{h S}$ are both individually small in which case there will be no observable dark force. In the second region, $\mu_{S}$ and $\mu_{h S}$ are large enough to give rise to an observable dark force but cancel against each other in Eq. (47) to maintain an ultralight mass. In the third region, as will become clear in later sections, $\mu_{S}$ and $\mu_{h S}$ are again individually small as in the first region, but each is determined by a sum of much larger terms that cancel among each other. The second region is phenomenologically the most interesting and is the focus of this paper.

\section{B. Nonrenormalizable interactions}

If both the $S$ and $H$ couple to additional fields with masses above the electroweak scale, then these interactions will in general induce nonrenormalizable operators that involve both the $S$ and $H$ in a low-energy effective theory that does not contain the heavy degrees of freedom explicitly. Minimal dark matter models, for example, can require TeV-scale DM particles in order to achieve the observed relic density [75], and these fields may generate the higher dimensional $S-H$ operators. At dimension five, one has seven independent operators coupling $S$ to the SM fermions and gauge bosons:

$$
\begin{aligned}
\mathcal{O}_{u}^{H} & =S \bar{Q}_{L} \epsilon H^{\dagger} C_{u}^{H} u_{R}+\text { H.c., } \\
\mathcal{O}_{d}^{H} & =S \bar{Q}_{L} H C_{d}^{H} d_{R}+\text { H.c., } \\
\mathcal{O}_{e}^{H} & =S \bar{L}_{L} H C_{e}^{H} e_{R}+\text { H.c., } \\
\mathcal{O}^{W} & =C^{W} S \operatorname{Tr}\left[W_{\mu \nu} W^{\mu \nu}\right], \\
\mathcal{O}^{B} & =C^{B} S B_{\mu \nu} B^{\mu \nu}, \\
\mathcal{O}^{G} & =C^{G} S \operatorname{Tr}\left[G_{\mu \nu} G^{\mu \nu}\right], \\
\mathcal{O}^{H} & =C^{H} S\left(H^{\dagger} H\right)\left(H^{\dagger} H\right) .
\end{aligned}
$$

The flavor indices on the fields $Q_{L}, L, u_{R}, e_{R}$ and the matrices $C_{u, d, e}^{H}$ are suppressed for simplicity. Operators of the form $S \bar{Q}_{L} i \not D Q_{L}, S \bar{u}_{R} i \not D u_{R}, S \bar{d}_{R} i \not D d_{R}$, and $S \bar{e}_{R} i \not D e_{R}$ can be related to the operators $\mathcal{O}_{u, d, e}^{H}$ by using the equations of motion

$$
\begin{aligned}
i \not D Q_{L} & =\epsilon H^{\dagger} Y_{u} u_{R}+H Y_{d} d_{R}, \\
i \not D u_{R} & =\epsilon H Y_{u}^{\dagger} Q_{L}, \\
i \not D d_{R} & =H^{\dagger} Y_{d}^{\dagger} Q_{L}, \\
i \not D e_{R} & =H^{\dagger} Y_{e}^{\dagger} L_{L},
\end{aligned}
$$

where $Y_{f}$ denotes the matrix of SM Yukawa couplings. We have omitted operators that involve derivative or pseudoscalar couplings of $S$. Such couplings are spin dependent and have a negligible effect in experiments which use unpolarized test objects.

In general, the Wilson coefficients $C_{u, d, e}^{H}$ are $3 \times 3$ matrices in flavor space, and can lead to flavor-changing interactions of quarks and leptons with $S$. Since the couplings of $S$ to quarks and leptons are extremely small (as dictated by the WEP violation bounds) there is no danger 
of introducing dangerous flavor-changing neutral currents. In the specific model examples considered in subsequent sections of the paper, find that the $C_{u, d, e}^{H}$ are proportional to the Yukawa matrices:

$$
C_{u, d, e}^{H} \equiv c_{u, d, e} Y_{u, d, e},
$$

where $c_{u, d, e}$ are the constants of proportionality. After expressing the fermion fields in the mass basis, in unitary gauge where the operators $\mathcal{O}_{u, d, e}^{H}$ become flavor diagonal. We can write

$\tilde{\mathcal{O}}_{u}^{H}=c_{u} y_{u}^{i} S \bar{u}_{L}^{i} H^{0} u_{R}^{i}+$ H.c. $\equiv \tilde{c}_{u}^{i} S \bar{u}_{L}^{i} H^{0} u_{R}^{i}+$ H.c.,

$\tilde{\mathcal{O}}_{d}^{H}=c_{d} y_{d}^{i} S \bar{d}_{L}^{i} H^{0} d_{R}^{i}+$ H.c. $\equiv \tilde{c}_{d}^{i} S \bar{d}_{L}^{i} H^{0} d_{R}^{i}+$ H.c.,

$\tilde{\mathcal{O}}_{e}^{H}=c_{e} y_{e}^{i} S \bar{e}_{L}^{i} H^{0} e_{R}^{i}+$ H.c. $\equiv \tilde{c}_{e}^{i} S \bar{e}_{L}^{i} H^{0} e_{R}^{i}+$ H.c.,

where $H^{0}$ is the lower component of the Higgs field $H$ in unitary gauge before electroweak symmetry breaking, the index $i=\{1,2,3\}$ runs over the three flavor generations, and we have defined $\tilde{c}_{a}^{i}=c_{a} y_{a}^{i}$.

After electroweak symmetry breaking, the operator $\mathcal{O}^{H}$ in Eq. (48) will also contribute to $\sin \theta$. To linear order in $h$ one has

$$
\mathcal{O}^{H} \rightarrow C^{H} v^{3} S h
$$

thereby generating a contribution to the off-diagonal element of the mass-squared matrix

$$
\Delta \mu_{h S}^{2}=2 C^{H} v^{3} .
$$

We will explore the consequences of this term when discussing scalar DM models below. For the moment we assume that this contribution has been included in $\sin \theta$.

Collecting the contributions to the couplings $g_{q, \ell, Q}$ from the higher-dimension operators $\mathcal{O}_{u, d, e}^{H}$ and mixing effects after electroweak symmetry breaking, the coupling of the ultralight scalar $\phi$ to SM fields at the electroweak scale is given by

$$
\begin{aligned}
g_{f}(v) & =\frac{m_{p}}{m_{f}}\left[\cos \theta \frac{v}{\sqrt{2}} \tilde{c}_{f}^{i}(v)-\sin \theta \frac{m_{f}}{v}(v)\right] \\
& \simeq \frac{m_{p}}{m_{f}} \frac{v}{\sqrt{2}} \tilde{c}_{f}(v)-\sin \theta \frac{m_{p}}{v}(v),
\end{aligned}
$$

where the last approximation is obtained from $\cos \theta \simeq 1$ since $\theta$ is constrained to be very small. We have included an extra factor of $m_{p} / m_{f}$ on the rhs above to be consistent with the convention in Eq. (15). We have ignored the running between the scales $\Lambda \sim \mathrm{TeV}$ and the electroweak scale for simplicity, but these effects can be incorporated by computing the appropriate anomalous dimension matrix and solving the corresponding renormalization group equations. We can now use Eq. (54) in Eqs. (23) and (25) to compute the Eötvös parameters. In particular, we note that the contribution proportional to $\sin \theta$ is universal, so its contribution to the $\eta_{S}$ can be evaluated using Eqs. (29) and (36).

In general, the origin and parametric dependence of $\sin \theta$ and $\tilde{c}_{f}$ are independent. In most of the parameter space where there are no strong cancellations between the two terms in Eq. (54), WEP violation constraints can separately bound each of the two terms in Eq. (54). In the next three sections we use this feature with the representative minimal DM models, in the presence of a dark force mediated by $\phi$, and determine the implications for terrestrial experiments of direct DM-detection, Eötvös experiments, and the colliders.

\section{Fine-tuning and the light scalar mass}

Before proceeding, we observe that in the absence of a symmetry that protects the light scalar mass from significant renormalization, one must resort to fine-tuning to maintain the long-range character of the dark force. To illustrate, we consider the contributions from the $H^{\dagger} H S$ and $H^{\dagger} H S^{2}$ in $V(H, S)$ to the singlet self-energy. In the unitary gauge one has

$$
\begin{aligned}
\Sigma\left(p^{2}\right)_{H^{\dagger} H S}= & -\frac{\delta_{1}^{2}}{128 \pi^{2}}\left[\frac{1}{\varepsilon}-\gamma+\ln 4 \pi+\ln \mu^{2}\right. \\
& \left.-F_{0}\left(m_{H}^{2}, m_{H}^{2}, p^{2}\right)\right] \\
\Sigma\left(p^{2}\right)_{H^{\dagger} H S^{2}}= & \frac{\delta_{2} m_{H}^{2}}{32 \pi^{2}}\left[\frac{1}{\varepsilon}-\gamma+1+\ln 4 \pi-\ln \frac{m_{H}^{2}}{\mu^{2}}\right]
\end{aligned}
$$

where we work in $d=4-2 \varepsilon$ dimensions, $\mu$ is the corresponding t'Hooft scale, and

$$
F_{0}(a, b, c)=\int_{0}^{1} d x \ln [(1-x) a+x b-x(1-x) c] .
$$

Had we regulated the integrals with a momentum cutoff $\Lambda_{\mathrm{CO}}$, the quadratic divergence proportional to $\delta_{2} m_{H}^{2} / \varepsilon$ would be replaced by an expression proportional to $\delta_{2} \Lambda_{\mathrm{CO}}^{2}$.

For either choice of regulator, preservation of a tiny scalar mass requires a mass counterterm $\delta \mu_{S}^{2}$ to cancel both the quadratic and logarithmically divergent contributions. In addition, as one sees using dimensional regularization, the finite, $\mu$-dependent contributions require a corresponding $\mu$-dependence in $\delta \mu_{S}^{2}$ as needed to maintain the scale-independence of the physical pole mass (or range of the dark force). The divergent and $\mu$-dependent finite contributions can be minimized by either choosing $\delta_{1,2}$ to be sufficiently tiny or by allowing for large cancellations between $\delta \mu_{S}^{2}(\mu)$ and the one-loop contributions. For the particular example discussed here, the finite contributions can also be minimized by choosing $\mu \approx m_{H}$, but these contributions will not be small at all scales unless the coefficients $\delta_{1,2}$ are tiny or there exists a large cancellation 
(fine-tuning) between $\delta \mu_{S}^{2}(\mu)$ and the one-loop contributions for $\mu \neq m_{H}$. In short, allowing for any appreciable interaction between the singlet $S$ and the Higgs sector of the SM invariably requires fine-tuning at some scale in order to ensure that the dark force mediator remains ultralight. In what follows, we will return to this point when considering the coupling of $S$ to DM. Even after allowing fine-tuning, the finite renormalized parameters are restricted in parameter space in order to maintain an ultralight mass for the dark force mediator. We will discuss this issue in more detail in Sec. VII.

\section{WIMP DM AND EÖTVÖS EXPERIMENTS}

In an earlier work [34], we examined constraints on the size of the coupling of an ultralight scalar to ordinary matter induced via virtual WIMP DM. The connection between constraints from galactic dynamics and Eötvös experiments and the size of the ultralight scalar couplings to DM and ordinary matter were shown in Fig. 1 of [34]. In analyzing the astrophysical constraints, we assumed only an upper bound $\beta<0.2$ and showed that in representative
WIMP scenarios, it leads to stronger constraints on the strength of the $\phi$-WIMP coupling than do the present Eötvös bounds on $\eta_{E \text {,DM }}$. An improvement [34] of about 8 orders of magnitude in Eötvös experiments would be required to compete with the bounds from astrophysical constraints.

Here, we explore the prospective implications of a nonvanishing $\beta$. The presence of a modified, long-range dark force could help alleviate tensions in the $\Lambda \mathrm{CDM}$ paradigm (we refer the reader to Refs. [33,51] for an extensive discussion). In what follows, we show that a nonvanishing $\beta$ implies a lower bound on $\eta_{E \text {,DM }}$ in simple WIMP scenarios, so that future Eötvös experiments with improved sensitivity could be used to test this possibility. For purposes of illustration, we consider both scalar and fermionic WIMP DM. For fermionic WIMPs we restrict our attention to vectorlike gauge representations, which simplifies the structure of the coupling of the ultralight singlet scalar $\phi$ to DM.

The Lagrangian for minimal WIMP DM takes the form

$$
\mathcal{L}= \begin{cases}\bar{\chi}\left(i \not D+M_{0}\right) \chi, & \text { fermionic DM } \\ c\left(D_{\mu} \chi\right)^{\dagger} D^{\mu} \chi-c M_{0}^{2} \chi^{\dagger} \chi-V(\chi, H), & \text { scalar DM }\end{cases}
$$

where $c=1 / 2$ for a real scalar and $c=1$ for a complex scalar. The covariant derivative depends on the $S U(2)_{L}$ and $U(1)_{Y}$ representations of $\chi$. Assuming that a single WIMP species saturates the relic density, one finds that typical masses of such minimal DM candidates are in the TeV range [75]. In general, $V(\chi, H)$ can contribute to the scalar DM mass after electroweak symmetry breaking. However, since the typical WIMP DM masses are in the TeV range, such a contribution which will be on the order of the electroweak scale, will be much smaller than the size of the mass parameter $M_{0} \sim \mathrm{TeV}$ in the second line of Eq. (58). Therefore in what follows, the total scalar DM mass, defined to be the sum of $M_{0}$ and the contribution to the mass term from $V(\chi, H)$ after electroweak symmetry breaking, is given entirely by $M_{0}$. For gauge singlet scalar DM models with DM masses in the $100 \mathrm{GeV}$ range, the contribution to the mass from electroweak symmetry breaking in $V(\chi, H)$ can be important. We will consider the case of singlet scalar DM in the next section. Furthermore, since electroweak symmetry breaking can in general induce mixing between the scalar DM and the Higgs, we impose a $Z_{2}^{\chi}$ symmetry $(\chi \rightarrow-\chi)$ to ensure stability of the DM particle. The interactions in $V(\chi, H)$ can also be constrained from WEP tests, and we will explore this in the next two sections for scalar DM. For vectorlike fermionic DM no renormalizable couplings exist between the Higgs and DM. Such couplings can however be present for chiral DM.

We consider the impact of a dark force via the interactions of DM with the ultralight scalar $\phi$ as in Eq. (12).
These couplings are gauge invariant for fermionic DM only for vectorlike gauge representations to which we restrict our attention. For chiral fermionic DM, the coupling to $\phi$ can only arise from higher-dimension operators by gauge invariance. Assuming no other low-energy degrees of freedom besides those of the SM plus the $\chi$ and $\phi$, the dark sector interactions (12) induce a coupling of $\phi$ to the SM fermions at two-loop order, as illustrated in Fig. 2. The graph involving only virtual $\mathrm{U}(1)_{Y}$ gauge bosons [left panel of Fig. 2] directly generate the operators $\mathcal{O}_{f}^{H}$ in Eq. (48), while the one-particle irreducible diagram involving both $W$ and $B$ bosons in the $\phi+f \rightarrow H+f$ "Compton amplitude" [right panel of Fig. 2] generates operators of the form $\bar{Q} i(\overleftarrow{\not D}-\overrightarrow{\not D}) Q$, etc. As noted earlier, operators of this type can be expressed in terms of $\mathcal{O}_{f}^{H}$ using the equations of motion, indicated symbolically by the presence of the $H$ field on the external leg in the right panel of Fig. 2. In either case, the Wilson coefficients $C_{f}^{H}$ are proportional to the Yukawa matrices due to the Higgs insertions. After the neutral component of the Higgs field obtains a vev, the loop-induced operators $\mathcal{O}_{f}^{H}$ give rise to the interactions $\bar{f} f \phi$ of Eq. (16). Any mixing between the ultralight scalar and the Higgs will also contribute, corresponding to the second term as usual in Eq. (54).

For $\mathrm{SU}(2)_{L}$ triplet $\mathrm{DM}$ with hypercharge $Y=0$, only the $\mathrm{SU}(2)_{L}$ gauge boson-exchange diagrams of the right panel of Fig. 2 contribute. The resulting coupling of $\phi$ to the SM fermions are as in Eq. (16), with 

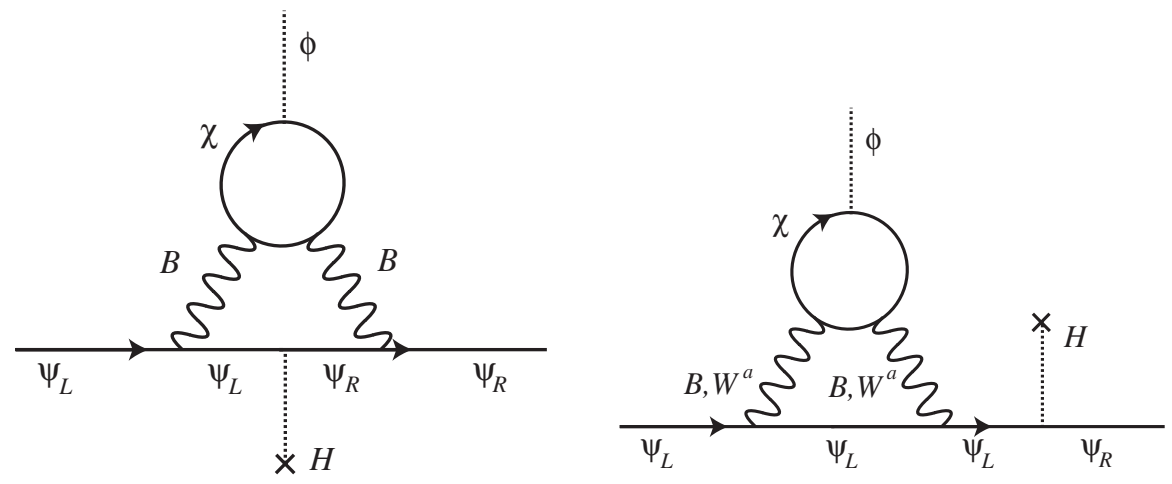

FIG. 2. Two-loop diagrams in WIMP DM models that generate the operators $\mathcal{O}_{f}^{H}$ in Eq. (48). Thus, after electroweak symmetry breaking the ultralight scalar couples to SM fermions.

$$
g_{f}=C_{3}\left(\frac{\alpha_{e m}}{\pi}\right)^{2} \frac{m_{p}}{M_{\chi}} g_{\chi} \hat{\xi}_{\chi}-\sin \theta \frac{m_{p}}{v},
$$

where we have employed naive dimensional analysis (NDA) to estimate the first term on the rhs of Eq. (59). Although the precise $\mathcal{O}(1)$ coefficient $C_{3}$ can be obtained from a complete computation, for our purposes of arriving at order-of-magnitude relationships between $\beta$ and $\eta$, the NDA expression suffices. ${ }^{5}$ We note that the sum of all loop graphs of the type in Fig. 2 is finite because we began with only renormalizable couplings and the operators $\mathcal{O}_{f}^{H}$ have dimension $n=5$. We also observe that the coupling to different species of fermions is universal since we have factored out the explicit dependence on the Yukawa coupling in the definition of the $g_{f}$ in Eq. (16).

For $\mathrm{SU}(2)_{L}$ multiplet $\mathrm{DM}$ with hypercharge $Y \neq 0$, the induced couplings of the ultralight scalar to DM is given by

$$
\begin{aligned}
g_{f}= & C_{N}\left(\frac{\alpha_{e m}}{\pi}\right)^{2} \frac{m_{p}}{M_{\chi}} g_{\chi} \hat{\xi}_{\chi}+C_{Y} Y^{2}\left(\frac{\alpha_{e m}}{4 \pi}\right)^{2} \frac{m_{p}}{M_{\chi}} g_{\chi} \hat{\xi}_{\chi} \\
& -\sin \theta \frac{m_{p}}{v}
\end{aligned}
$$

where $C_{N, Y}$ are $\mathcal{O}(1)$ coefficients that, as before, can be obtained from a complete two-loop computation. We observe that the first terms on the right-hand side of Eqs. (59) and (60) are universal for different fermion species and come from the exchange of the $S U(2)_{L}$ gauge bosons $W^{a}$ in Fig. 2. The last terms containing $\sin \theta$ are also universal, having been generated from the mixing between the Higgs and light scalar $\phi$. The middle term in Eq. (60), involving the square of the SM hypercharge $Y$, are nonuniversal and are generated by the exchange of $\mathrm{U}(1)_{Y}$ gauge-boson $B$. We point out that such minimal WIMP DM models with nonzero hypercharge are typically ruled out [75] by directdetection experiments. Here we discuss these minimal DM models with nonzero hypercharge, only as illustrative examples keeping in mind that such DM could be part of a

\footnotetext{
${ }^{5}$ The subscript in $C_{3}$ refers to the dimension of the triplet representation of $\mathrm{SU}(2)_{L}$.
}

nonminimal extension which avoids the direct-detection bounds.

A similar analysis can be performed for other WIMP models of DM that may involve additional degrees of freedom. In supersymmetry, for example, the DM matter particle $\chi$ is a linear superposition of winos, binos, and Higgsinos. In addition there are squark and slepton particles which give interactions of the type $\lambda \tilde{\psi} \bar{\psi} \chi+$ H.c.. In theories with such a spectrum of particles one can induce a coupling of $\phi$ to ordinary matter via virtual DM at one loop as shown in Fig. 3. ${ }^{6}$ If the ultralight scalar $\phi$ is the scalar component of a singlet superfield $\hat{S}$, a superpotential term of the form $\left(\mu+g_{\chi} \hat{S}\right) \hat{H}_{u} \cdot \hat{H}_{d}$ will lead to a coupling to fermions of the form

$$
g_{f} \sim \frac{1}{16 \pi^{2}} \frac{m_{\tilde{\psi}} \mu \lambda^{2}}{M_{\mathrm{SUSY}}^{2}} g_{\chi} .
$$

If $\chi$ is primarily a bino, then $\lambda \simeq g_{Y}$, the hypercharge coupling. If $\chi$ is primarily Higgsino, the coupling of $\phi$ to the light quarks will be suppressed. The coupling of $\phi$ will be primarily to the top quark which has order one Yukawa couplings. Thus, in such models it is possible to induce a stronger WEP-violating coupling to ordinary matter at one loop leading to bigger effects in Eötvös experiments. For the sake of brevity, we do not consider such nonminimal scenarios and we will only focus on minimal DM models without additional degrees of freedom such as squarks and sleptons.

It is possible that the loop-induced $\mathcal{O}_{f}^{H}$ operator contributions to $g_{f}$ and those generated indirectly by $H-S$ mixing (proportional to $\sin \theta$ ) are individually much larger than $g_{f}$ yet cancel to produce a much smaller coupling. However, away from this special region of parameter

\footnotetext{
${ }^{6}$ Of course the presence of an ultralight scalar would introduce a new hierarchy problem which spoils the main motivation for supersymmetric theories. Here we invoke supersymmetry simply as a familiar example to illustrate the possibility of new types of interactions that can induce a coupling of the ultralight scalar to ordinary matter.
} 


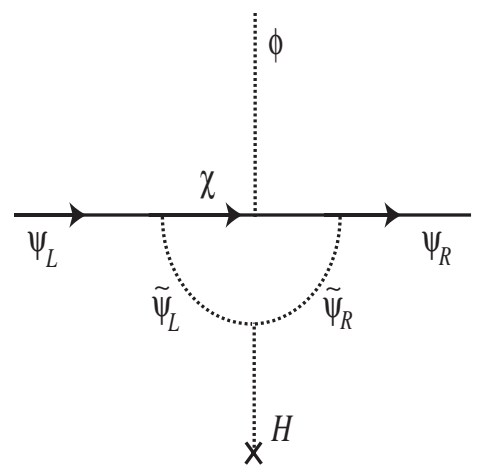

FIG. 3. DM-induced coupling of $\phi$ to SM fermions at one loop in the presence of additional squark and slepton like degrees of freedom.

space, each contribution will be roughly no larger in magnitude than $g_{f}$ itself [as already discussed following Eq. (54)]. In this generic case, we are able to obtain expectations for the size of Eötvös parameters in our illustrative minimal WIMP dark sector models, for a given value of $\beta$, from the two-loop gauge contribution. We will consider the contribution of $H-S$ mixing to $g_{f}$ in the next section. We note that the contributions from the $\mathrm{SU}(2)_{L}$ gauge bosons are generically an order-of-magnitude larger than those from the hypercharge gauge bosons due to the relative sizes of their couplings [leading to the additional factor of $1 / 16$ in the second term of Eq. (60)]. Consequently, for purposes of making order-of-magnitude estimates, we may employ the expressions for $\eta_{S}$ in the presence of universal couplings given in Eqs. (29) and (36) with

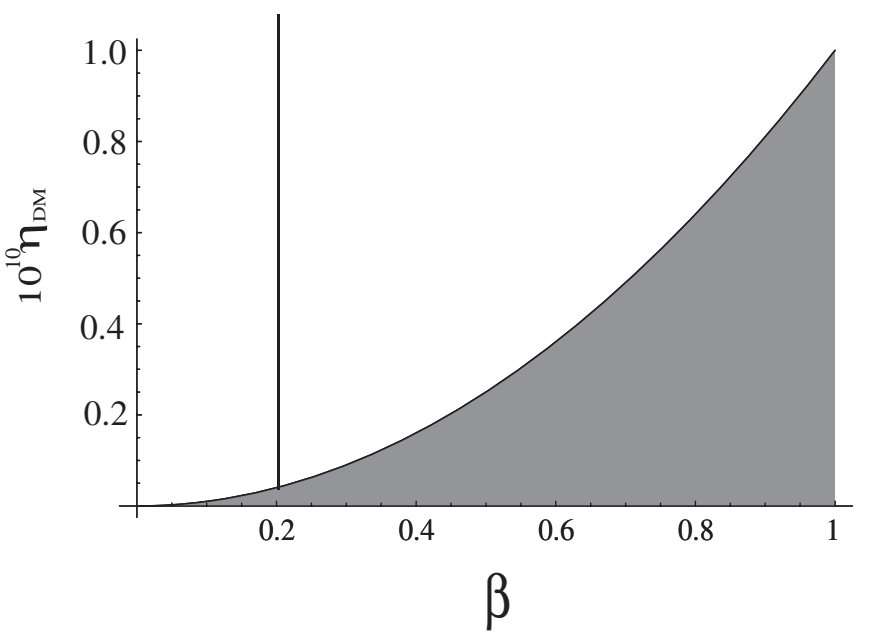

$$
\bar{g} \rightarrow\left(\frac{\alpha_{e m}}{\pi}\right)^{2} \frac{m_{p}}{M_{\chi}} g_{\chi} \hat{\xi}_{\chi}
$$

Expressing $g_{\chi} \hat{\xi}_{\chi}$ in terms of $\beta$ we then obtain

$$
\begin{aligned}
\frac{\eta_{\mathrm{DM}}}{\beta^{2}} \gtrsim\left(\frac{7}{9}\right)\left(\frac{\alpha}{\pi}\right)^{2} & \left|\left(\frac{Z_{1}}{A_{1}}-\frac{Z_{2}}{A_{2}}\right)\left\{\frac{m_{e}}{m_{N}}+\sum_{q} \frac{m_{q}}{m_{N}}\left(x_{q, p}-x_{q, n}\right)\right\}\right| \\
\frac{\eta_{E}}{\beta^{2}} \gtrsim & \left(\frac{7}{9}\right)\left(\frac{\alpha}{\pi}\right)^{4}\left(\frac{v}{m_{N}}\right) \mid\left(\frac{Z_{1}}{A_{1}}-\frac{Z_{2}}{A_{2}}\right) \\
& \times\left\{\frac{m_{e}}{m_{N}}+\sum \frac{m_{q}}{m_{N}}\left(x_{q, p}-x_{q, n}\right)\right\} \mid \\
& \times\left[\frac{g_{h}\left(N_{p}+N_{n}\right)+\left(m_{e} / v\right) N_{e}}{\left(N_{p}+N_{n}\right)+\left(m_{e} / m_{N}\right) N_{e}}\right] .
\end{aligned}
$$

Numerically the bounds in Eqs. (63) and (64) are (for Be and Ti laboratory samples with $\left|Z_{1} / A_{1}-Z_{2} / A_{2}\right| \simeq 1 / 72$ )

$$
\frac{\eta_{\mathrm{DM}}}{\beta^{2}} \gtrsim 10^{-10}, \quad \frac{\eta_{E}}{\beta^{2}} \gtrsim 10^{-16},
$$

which are shown in Fig. 4 as the allowed regions for typical minimal WIMP DM models in the $\left(\eta_{\mathrm{DM}, E}, \beta\right)$ parameter space. The curve in the left and right figures gives as estimate of the minimum size for $\eta_{\mathrm{DM}}$ and $\eta_{E}$ respectively as a function of $\beta$. One can also estimate the ratio $\eta_{E} / \eta_{\mathrm{DM}}$ from Eqs. (63) and (64) to be approximately

$$
\eta_{E} / \eta_{\mathrm{DM}} \simeq 10^{-6}
$$

For $\beta=0.2$, marked by the vertical lines in Fig. 4, the current upper bound from galactic dynamics [51], we see

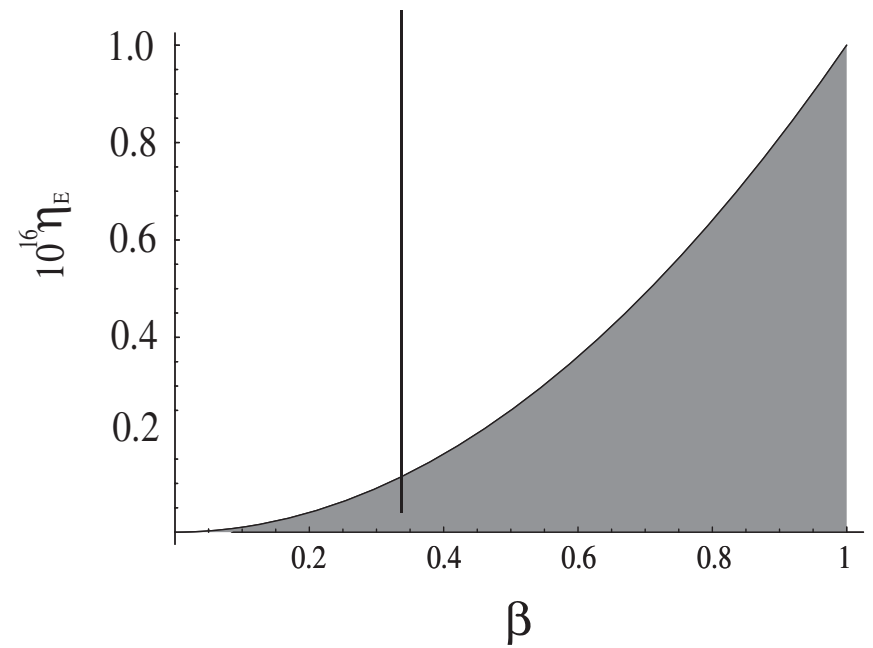

FIG. 4. An estimate of the allowed region in the $\left(\eta_{\mathrm{DM}, E}, \beta\right)$ parameter space for minimal WIMP DM models. The curves in the figures give an estimate of for $\eta_{\mathrm{DM}, E}$ for a given value of $\beta$ from the two-loop diagrams in Fig. 2 . The shaded region is unlikely for typical WIMP models. Using the observational constraint $\beta<0.2$, the allowed region is further restricted to the left of the vertical line. The estimates in the above figures for $\eta_{\mathrm{DM}, E}$, for $\beta<0.2$, are far below the current experimental bounds $\eta_{\mathrm{DM}} \lesssim 10^{-5}, \eta_{E} \lesssim 10^{-13}$. An improvement of about 5 orders of magnitude would be required in Eötvös experiments to fully probe the allowed parameter space for $\beta=0.2$ by measuring $\eta_{E}$. This is within reach of the MiniSTEP [56] proposal. 
that the lower bounds for typical WIMP DM models are $\eta_{\text {DM }}>4 \times 10^{-12}$ and $\eta_{E}>4 \times 10^{-18}$. These lower bounds are far below the current experimental upper bounds shown in Eq. (10). An improvement of about five to 7 orders of magnitude in Eötvös experiments would be required in order to probe these expectations of WIMP DM models. The MiniSTEP [56] experiment, which is currently under study, is expected to reach a sensitivity for $\eta_{E}$ of about $10^{-18}$ and might be able to probe the lower bounds of these WIMP models. However, if $\beta<0.05$ as indicated by a recent analysis [54] of the CMB and large scale structure formation, the lower bounds on $\eta_{\mathrm{DM}, E}$ are far beyond current and future planned experiments. If an effect is detected in $\eta_{\text {DM }, E}$ far above the expectations in Fig. 4 it would suggest the possibility that the coupling of $\phi$ to the SM fermions is mostly via $h-\phi$ mixing corresponding to the last term appearing in Eqs. (59) and (60). One could extract a value for $\sin \theta$ and derive implications for various DM scenarios as discussed in the following section. Another possibility that might explain an effect above the expectation in Fig. 4 would be a stronger induced coupling of $\phi$ to ordinary matter in nonminimal DM models, for example, a one-loop coupling of $\phi$ to ordinary matter (see Fig. 3) in the presence of additional squark degrees of freedom.

\section{WEP TESTS, DIRECT DETECTION, AND HIGGS BOSON DECAYS}

As observed in Ref. [33], the presence of a nonvanishing $\beta$ of astrophysically interesting magnitude, together with present limits on $\eta_{E \text {,DM }}$ can imply upper bounds on the size of DM-nucleus cross sections relevant for direct-detection experiments. Here we analyze these bounds in detail for the illustrative cases of scalar DM scenarios and argue that upper bounds on the DM-nucleus cross sections are less stringent than obtained in Ref. [33]. We further comment on the analysis of Ref. [33] at the end of Sec. VII. We also consider the implications of a dark force for the DM relic density and derive corresponding constraints. Finally, using a light scalar triplet, as part of a multicomponent DM scenario, we show how the presence of a nonvanishing $\beta$ together with experimental limits on $\eta_{E \text {,DM }}$ - can preclude observable shifts in the rate for the Higgs boson to decay to two photons as one might otherwise expect.

To include the full set of possible renormalizable interactions between the DM, SM fields, and ultralight scalar, we expand the scalar potential of Eq. (38), imposing the $Z_{2}^{X}$ $(\chi \rightarrow-\chi)$ symmetry need to prevent DM decays:

$$
\begin{aligned}
V(H, S, \chi)= & V(H, S)+\frac{1}{2} M_{0}^{2} \chi^{2}+\frac{\lambda_{\chi}}{4} \chi^{4} \\
& +a_{2} H^{\dagger} H \chi^{2}+g_{\chi} \chi^{2} S+\lambda_{\chi s} \chi^{2} S^{2} .
\end{aligned}
$$

For the scalar singlet case, $\chi$ is a real field, while for the real triplet with components $\chi^{0}$ and $\chi^{ \pm}$, one has [76]

$$
\chi^{2}=\left(\chi^{0}\right)^{2}+2 \chi^{+} \chi^{-} .
$$

We take $M_{0}^{2}$ and $a_{2}$ to be positive in order prevent a nonvanishing vev for $\chi$ and the occurrence of phenomenologically unacceptable cosmological domain walls. The experimental constraints on this DM model, for $g_{\chi}=0$, were recently explored in $[57,58]$.

After electroweak symmetry breaking, the $H^{\dagger} H \chi^{2}$ term generates a contribution to the DM mass:

$$
M_{\chi}^{2}=M_{0}^{2}+a_{2} v^{2} .
$$

Henceforth, we will take $v=246 \mathrm{GeV}, M_{\chi}^{2}, a_{2}$, and the mass of the SM-like Higgs boson $\left(m_{h}\right)$ as independent parameters. All of them govern the $\chi$-nucleus cross section, whose leading order amplitude is generated by $t$-channel Higgs exchange as in Fig. 5 and is given by

$$
\mathcal{M} \simeq \frac{2 a_{2} g_{h} v}{m_{h}^{2}} \bar{N} N,
$$

where we have neglected the $t$-dependence of the amplitude for simplicity. Note that since the real triplet has zero hypercharge, the elastic DM-nucleus scattering has no contribution form $Z$-boson exchange at tree level. The corresponding cross section is

$$
\sigma_{\chi N} \simeq \frac{a_{2}^{2} g_{h}^{2} v^{2} m_{N}^{2}}{\pi\left(M_{\chi}+m_{N}\right)^{2} m_{h}^{4}},
$$

where, for simplicity, we have dropped the dependence on momentum transfer to the nucleus. [Recall that $g_{h} \simeq$ $1.71 \times 10^{-3}$ is the coupling of the Higgs to the nucleon as defined in Eq. (34)]. Note that the cross section decreases for increasing $M_{\chi}$ or decreasing $a_{2}$. Note also that the coupling $a_{2}$, together with the masses $M_{\chi}$ and $m_{h}$,

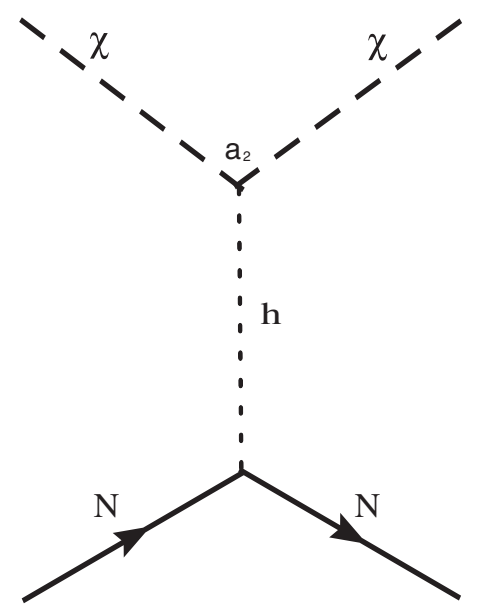

FIG. 5. Direct-detection process for the scalar singlet and triplet $\chi$ via t-channel Higgs exchange with a nucleon. The magnitude of the detection rate is determined by the strength of the coupling $a_{2}$. If $\chi$ couples to the ultralight scalar $\phi$, the size of $a_{2}$ and thus the detection rate is bound by WEP violation constraints. 


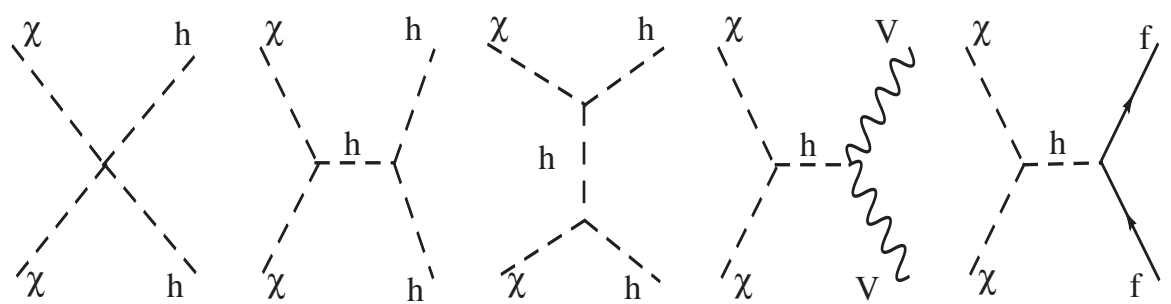

FIG. 6. Annihilation diagrams for the scalar singlet DM. For the scalar triplet DM one has in addition the usual annihilation diagrams mediated by gauge interactions. If the gauge interactions of the triplet dominate the dynamics of annihilation, a DM mass of around $2 \mathrm{TeV}$ is needed to saturate the relic density.

control the $\chi$ relic density through the annihilation diagrams of Fig. 6. For $M_{\chi} \sim m_{h} / 2$ for singlet DM or a light triplet in the multicomponent DM scenario, the Higgsexchange contribution becomes large, requiring a suppression of $a_{2}$ in order to maintain the observed CDM relic density. In what follows, we will generally avoid this regime.

\section{A. WEP Tests and Ultralight-Scalar-Higgs mixing}

A relation between the Higgs-exchange contribution to $\sigma_{\chi N}$ and $\eta_{E, \mathrm{DM}}$ arises for nonvanishing $\beta$ because the parameters $a_{2}, M_{\chi}$, and $m_{h}$ that enter the cross section also control the strength of the DM-loop induced mixing between the Higgs boson and the ultralight scalar. After electroweak symmetry-breaking, these loop effects generate contributions to the mass-squared parameters $\mu_{h s}^{2}$ and $\mu_{S}^{2}$. The parameter $\mu_{S}^{2}$ contributes only to the mass $m_{\phi}^{2}$ and $\mu_{h S}^{2}$ contributes to $\sin \theta$ and $m_{\phi}^{2}$. As with the contributions from Higgs loops to $\mu_{S}^{2}$ discussed earlier in Sec. IV C, the DM-loop contributions to this mass-squared parameter will also require the introduction of fine-tuning to maintain a sufficiently small mass for the ultralight scalar. Furthermore, as already mentioned and discussed in more detail in Sec. VII, the finite renormalized parameter $\mu_{S}^{2}$ must be further restricted in parameter space in order to maintain $m_{\phi}<10^{-25} \mathrm{eV}$ along with a dark force large enough to be observed. We implicitly assume that we are in this region of parameter space, conducive to the obser- vation of a long-range dark force. We will discuss the implications of other regions in parameter space in Sec. VII.

We begin by observing that in addition to the direct coupling $\delta_{1}$ of $S$ to the Higgs via the operator $H^{\dagger} H S$, a DM-induced $\phi$-matter coupling arises from the one-loop contribution to this operator through the second diagram of Fig. 7. After renormalization in the $\overline{\mathrm{MS}}$ scheme, the resulting finite coefficient of his operator is

$$
\delta_{1}^{\text {ren }}=\delta_{1}(\mu)+\kappa \frac{g_{\chi} a_{2}}{4 \pi^{2}} \ln \frac{M_{0}^{2}}{\mu^{2}},
$$

where

$$
\kappa= \begin{cases}1, & \text { singlet } \chi \\ 3, & \text { triplet } \chi\end{cases}
$$

The factor of $\kappa=3$ appears in the case of the triplet $\chi$ due to the three components of the triplet traversing the loop in the second diagram of Fig. 7. Here $\delta_{1}(\mu)$ is the finite, scale-dependent coupling counterterm whose numerical value is a priori unknown and whose presence is required to ensure renormalization group ( $R G)$ invariance of the physical properties of the $\phi$ and $h$. Note that the mass parameter $M_{0}^{2}$ (taken here to be positive) rather than $M_{\chi}^{2}$ appears in the argument of the logarithm since we are working in the theory before electroweak symmetrybreaking.
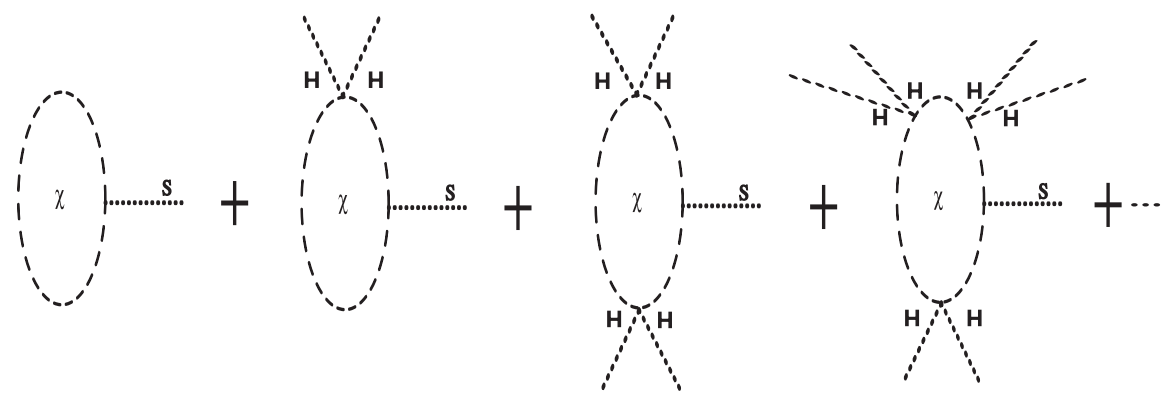

FIG. 7. One-loop diagrams which contribute to the effective potential $V(H, S)$ with one external $S$ field. After electroweak symmetry breaking the effective potential contributes to Higgs-ultralight-scalar mixing. The first two diagrams are UV divergent and contribute to the renormalization of the $S$-tadpole and the coupling $\delta_{1}$ respectively. The remaining diagrams mix into higher dimensional operators and give a finite contribution to Higgs-ultralight-scalar mixing as explained in Appendix B. 
We also observe that the $\chi^{2} S$ interaction will generate a contribution to the mass parameter $\mu_{S}^{2}$ as it yields the nonvanishing contribution to the $S$ self energy:

$$
\begin{aligned}
\Sigma\left(p^{2}\right)_{\chi{ }^{2} S}= & -\frac{g_{\chi}^{2}}{16 \pi^{2}}\left[\frac{1}{\varepsilon}-\gamma+\ln 4 \pi+\ln \mu^{2}\right. \\
& \left.-F_{0}\left(M_{0}^{2}, M_{0}^{2}, p^{2}\right)\right] .
\end{aligned}
$$

As with the case of the logarithmically divergent Higgs contribution $\Sigma\left(p^{2}\right)_{H^{\dagger} H S}$ of Eq. (55), the DM-loop contribution to the self energy requires a corresponding $\mu$-dependence in $\delta \mu_{S}^{2}(\mu)$ to maintain RG invariance of the pole mass that governs the range of the dark force. Large DM-loop contributions to $\mu_{S}^{2}$ can be minimized at all scales by taking $g_{\chi}$ to be sufficiently small: $g_{\chi} \lesssim$ $4 \pi m_{S}^{\text {pole }}$. Doing so, however, would preclude a value of $\beta$ of astrophysically relevant strength. Alternatively, one may allow for a much larger, phenomenologically interesting magnitude for $g_{\chi}$ and maintain a small $\mu_{S}^{2}$ by invoking fine-tuning between the one-loop contribution of Eq. (74) and $\delta m^{2}(\mu)$.

A similar set of alternatives applies to the renormalized coupling $\delta_{1}^{\text {ren }}$. One could require that the product $g_{\chi} a_{2}$ be sufficiently small in magnitude, with a correspondingly tiny $\delta_{1}(\mu)$, so that the induced $H-S$ mixing is consistent with the present bounds on $\eta_{E \text {,DM }}$. To obtain a large $\beta$, one must then take $a_{2}$ to be sufficiently small, implying an upper bound on the Higgs-exchange contribution to the DM-nucleus cross section via Eq. (71). This choice is essentially the strategy followed in Ref. [33] to obtain upper bounds on $\sigma_{\chi N}$. However, as seen in Eq. (41), $\delta_{1}$ contributes to $\mu_{h S}$ and thus to the mass $m_{\phi}$ via Eq. (47). The condition of $m_{\phi}<10^{-25} \mathrm{eV}$ gives a much stronger naturalness constraint on $a_{2}$ forcing it to be essentially zero for a nonzero dark force. The constraints from $\eta_{E \text {,DM }}$ are thus not relevant in such a naturalness analysis. We will also discuss this in more detail in Sec. VII.

In what follows, we will instead allow for fine-tuning in both $\delta_{1}$ since we have already allowed fine-tuning for $\mu_{S}$. We show that assuming $\mu_{S}^{2}$ is restricted in parameter space to satisfy $m_{\phi}<10^{-25} \mathrm{eV}$ for any value of $\mu_{h S}^{2}$ in Eq. (47), we can obtain upper bounds on $\sigma_{\chi N}$ by analyzing finite, one-loop contributions to $\mu_{h S}^{2}$, from higher dimensional operators after EWSB, and their implications for WEP tests. The other regions in parameter space and their implications will be discussed in Sec. VII. To that end, consider the third diagram of Fig. 7, which generates a contribution to the dimension five operator

$$
C_{2}\left(H^{\dagger} H\right)\left(H^{\dagger} H\right) S,
$$

where in the coefficient $C_{2}$ is finite and given by

$$
C_{2}=\kappa \frac{a_{2}^{2}}{8 \pi^{2}} \frac{g_{\chi}}{M_{0}^{2}} .
$$

Since this contribution is finite there is no counterterm involved in determining the value of $C_{2}$. After electroweak symmetry breaking this term will generate a contribution to the off-diagonal elements in the $h-S$ mass-squared matrix

$$
\mu_{h S}^{2}=2 C_{2} v^{3}+\delta_{1} v,
$$

leading to an $h-S$ mixing angle $\theta$

$$
\tan \theta=\frac{x}{1+\sqrt{1+x^{2}}}, \quad x=\frac{\mu_{h S}^{2}}{\mu_{h}^{2}}=\frac{2 C_{2} v^{3}+\delta_{1} v}{m_{h}^{2}},
$$

which was defined in Eqs. (42) and (45). Since this mixing implies a coupling of $\phi \approx S$ to matter, the loop-induced coefficient $C_{2}$ will contribute to the Eötvös parameters $\eta_{\mathrm{DM}, E}$. Given the dependence of $C_{2}$ on $a_{2}$ and the absence of any fine-tuning in this parameter, we obtain an upper bound on $\sigma_{\chi N}$ for nonvanishing $\beta$ as described below.

Before doing so, we observe the contribution to $\mu_{h S}^{2}$ from full series of diagrams appearing in Fig. 7 (plus the tadpole graph generated by the $\chi^{2} S$ interaction) can be evaluated in a straightforward way as outlined in Appendix B. After renormalization, the result is

$$
\mu_{h S}^{2}=v\left[\delta_{1}(\mu)+\kappa \frac{g_{\chi} a_{2}}{4 \pi^{2}}\left(\ln \frac{M_{\chi}^{2}}{\mu^{2}}-1\right)\right]+\kappa \frac{g_{\chi} a_{2}^{2}}{4 \pi^{2}} \frac{v^{3}}{M_{\chi}^{2}} .
$$

Apart from an overall constant in the first term and the replacement $M_{0} \rightarrow M_{\chi}$, this expression is the same as we obtained using the contributions to the $H^{\dagger} H S$ and $\left(H^{\dagger} H\right)^{2} S$ operators from the second and third diagrams of Fig. 7. The expression in Eq. (79) has the advantage that it depends on the tree-level $\chi$ mass after electroweak symmetry breaking rather than on the parameter $M_{0}$ as in the effective operator analysis. We will henceforth use the finite, second term in Eq. (79) to derive an upper bound on Higgs-exchange contributions to $\sigma_{\chi N}$.

To that end, we write the mixing angle as

$$
\begin{aligned}
\sin \theta & \approx \tan \theta \approx x \approx \kappa \frac{a_{2}^{2}}{4 \pi^{2}} \frac{g_{\chi} v^{3}}{M_{\chi}^{2} m_{h}^{2}}+\frac{\delta_{1}^{\mathrm{ren}} v}{m_{h}^{2}} \\
& =\kappa \frac{a_{2}^{2}}{\pi^{3 / 2}} \frac{v^{3}}{M_{P} m_{h}^{2}} \beta+\frac{\delta_{1}^{\mathrm{ren}} v}{m_{h}^{2}},
\end{aligned}
$$

where $\delta_{1}^{\text {ren }}$ denotes the quantity in square brackets in Eq. (79). The mixing angle $\sin \theta$ also characterizes the universal $H-S$ mixing contribution to the Eötvös parameters $\eta_{E \text {,DM }}$. We now require that the contribution from each term on the right-hand side of Eq. (80) to $\eta_{E \text {,DM }}$ be no larger than the experimental limits on these parameters. As discussed previously, the different parametric dependence of each term and avoiding slices of parameter space with unnatural cancellations between the two allows us to treat each one separately. Considering only the first term pro- 
portional to $a_{2}^{2}$, using Eqs. (29) and (36), with

$$
\bar{g} \rightarrow-\sin \theta \frac{m_{N}}{v}
$$

and expressing $g_{\chi}$ in terms of $\beta$, we obtain

$$
\begin{aligned}
\frac{\eta_{\mathrm{DM}}}{\beta^{2}} \simeq & a_{2}^{2}\left(\frac{7 \kappa}{18 \pi}\right)\left(\frac{v}{m_{h}}\right)^{2} \\
& \times\left|\left(\frac{Z_{1}}{A_{1}}-\frac{Z_{2}}{A_{2}}\right)\left\{\frac{m_{e}}{m_{N}}+\sum_{q} \frac{m_{q}}{m_{N}}\left(x_{q, p}-x_{q, n}\right)\right\}\right|
\end{aligned}
$$
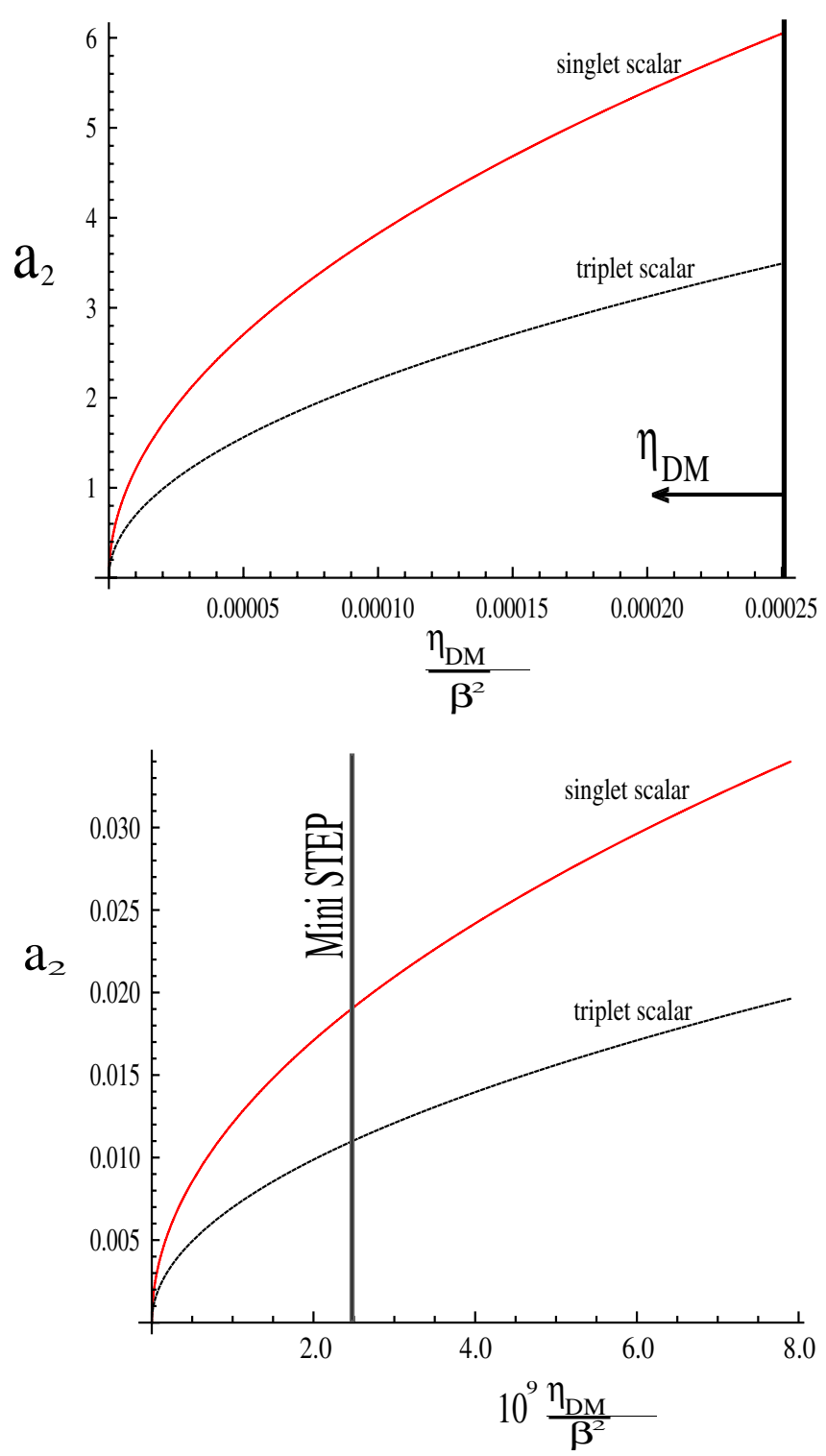

$$
\begin{aligned}
\frac{\eta_{E}}{\beta^{2}} \simeq & a_{2}^{4}\left(\frac{7 \kappa}{36 \pi^{4}}\right)\left(\frac{v}{m_{h}}\right)^{4}\left(\frac{v}{m_{N}}\right) \\
& \times\left|\left(\frac{Z_{1}}{A_{1}}-\frac{Z_{2}}{A_{2}}\right)\left\{\frac{m_{e}}{m_{N}}+\sum_{q} \frac{m_{q}}{m_{N}}\left(x_{q, p}-x_{q, n}\right)\right\}\right| \\
& \times\left[\frac{g_{h}\left(N_{p}+N_{n}\right)+\left(m_{e} / v\right) N_{e}}{\left(N_{p}+N_{n}\right)+\left(m_{e} / m_{N}\right) N_{e}}\right] .
\end{aligned}
$$

Equations (82) and (83) imply that for fixed $\beta$ and $m_{h}$, the experimental bounds on $\eta_{E \text {,DM }}$ translate into bounds on $a_{2}$ as shown in Fig. 8. The solid red curves and the dashed
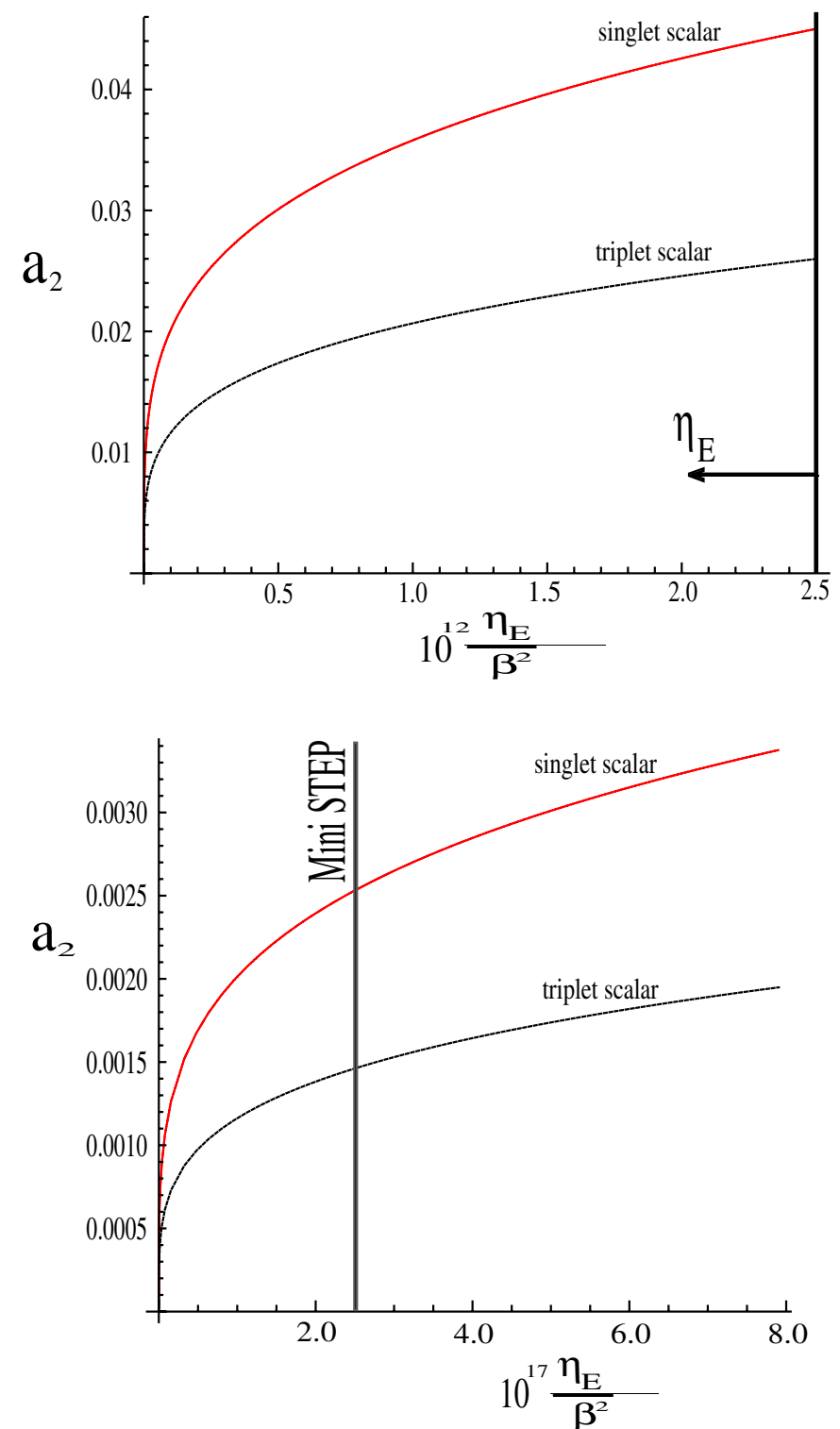

FIG. 8 (color online). Upper bound on $a_{2}$ in the singlet (red-solid) and real triplet (black-dotted) scalar DM models as a function of $\eta_{\mathrm{DM}} / \beta^{2}$ (left panel) and $\eta_{E} / \beta^{2}$ (right panel). We have used $m_{h}=120 \mathrm{GeV}$ and assume $\beta=0.2$ to discuss the resulting bounds on $a_{2}$ from Eötvös experiments. In the top left and right plots, the vertical black lines on the right correspond to the upper bounds $\eta_{\mathrm{DM}}<$ $10^{-5}$ and $\eta_{E}<10^{-13}$ respectively. These vertical black lines will move to the left with further improvements in Eötvös experiments as indicated by the left-pointing arrow in each plot. The bottom left and right plots show the region closer to the expected future bounds, from the MiniSTEP experiment, of $\eta_{\mathrm{DM}}<10^{-10}$ and $\eta_{E}<10^{-18}$ respectively as indicated by the vertical black line in each plot. We explore the implications of these bounds on $a_{2}$ from Eötvös experiments for specific observables in Secs. VIC and VID. 
black curves correspond to the bounds on $a_{2}$ in the singlet $\chi$ and real triplet $\chi$ models, respectively. For $\beta=0.2$ the current bounds in Eötvös experiments imply $\eta_{\mathrm{DM}} / \beta^{2}<$ $2.5 \times 10^{-4}$ and $\eta_{E} / \beta^{2}<2.5 \times 10^{-12}$ respectively. The tighter bound from $\eta_{E}$ implies $a_{2}<0.042$ and $a_{2}<$ 0.026 for the singlet and triplet $\chi$ models, respectively. The possible future experiment like MiniSTEP is expected to improve the sensitivity of $\eta_{\mathrm{DM}, E}$ by 5 orders of magnitude. From Eq. (83) this would tighten the bound on $a_{2}$ by an additional factor of $10^{5 / 4}$ for a nonzero $\beta$ or lead to the discovery of a dark force effect.

\section{B. WEP Tests and Scalar singlet DM Relic density}

For the scalar singlet DM, the DM relic density is determined entirely by the parameter $a_{2}$ for fixed DM and Higgs masses. This feature can be seen from Fig. 6, where $a_{2}$ enters the amplitude for each annihilation diagram and, thus, determines the DM annihilation rate. In particular, the value of $a_{2}$ must be sufficiently large so that the DM relic density does not over saturate the observed value. Thus, the requirement that the singlet $\mathrm{DM}$ relic density smaller than the total DM relic density, $\Omega_{\mathrm{DM}}^{S} \leq$ $\Omega_{\mathrm{DM}}$, leads to a minimum value $a_{2}^{\min }$ for fixed values of $M_{\chi}$ and $m_{h}$. If the WEP bounds on $a_{2}$ imply that $a_{2}<a_{2}^{\min }$, then a dark force of the corresponding strength will be ruled out.

In order to illustrate this interplay, we refer to Fig. 3 of [58]. The parameters $a_{2}$ and $M_{\chi}$ are the same as $\lambda$ and $m_{D}$ respectively in the notation of [58]. From Fig. 3 of that work, we see that for DM masses in the $0-50 \mathrm{GeV}$ range, the required value of $a_{2}$ ranges from $\sim 0.16-0.05$ respectively for $m_{h}=120 \mathrm{GeV}$. On the other hand, from the upper curve in the top right graph of Fig. 8, we see that $a_{2} \lesssim 0.045$ for $\beta=0.2$ from the current bound of $\eta_{E}<$ $10^{-13}$ (vertical black line at right). This WEP constraint $a_{2}<0.045$ implies an overdensity of DM in the range $0<$ $M_{\chi}<50 \mathrm{GeV}$ thus ruling out the possibility of a dark force with $\beta>0.2$ in this range of parameter space. We give sample points in the parameter space of singlet DM models in Table II.

TABLE II. The first two columns give sample points in the $\left(a_{2 \text { relic }}, M_{\chi}\right)$ space of scalar singlet DM models with a Higgs mass of $m_{h}=120 \mathrm{GeV}$. The third column gives an expectation for $\eta_{E} / \beta^{2}$ from Eq. (83). The fourth column uses the current bound of $\eta_{E}<10^{-13}$ to determine whether a dark force of $\beta=$ 0.2 is ruled out. One can equivalently compare the different values of $a_{2 \text { relic }}$ with the WEP bound on $a_{2}$, at $\beta=0.2$, in top right graph of Fig. 8 at the far right vertical line.

\begin{tabular}{lccc}
\hline \hline$a_{2 \text { relic }}$ & $M_{\chi}(\mathrm{GeV})$ & Expectation for $\frac{\eta_{E}}{\beta^{2}}$ & $\beta=0.2$ \\
\hline 0.15 & 20 & $4 \times 10^{-10}$ & Excluded \\
0.10 & 40 & $7 \times 10^{-11}$ & Excluded \\
0.02 & 100 & $1 \times 10^{-13}$ & Allowed \\
\hline \hline
\end{tabular}

A more detailed analysis can be performed to rule out even smaller values of $\beta$ depending on the DM mass in the scalar singlet model. Future Eötvös experiments with the sensitivity of MiniSTEP [56] which are expected to reach a sensitivity of $\eta_{E}<10^{-18}$, could require bound of $a_{2} \lesssim$ 0.0025 for $\beta=0.2$ as seen in the bottom right graph of Fig. 8. In this case, one can rule out $\beta<0.2$ even for DM masses above $60 \mathrm{GeV}$ which require smaller values of $a_{2}$ in order to get the right relic density. As seen in Fig. 3 of [58], larger values of the Higgs mass typically imply much larger values of $a_{2}$. For example, a Higgs mass of $200 \mathrm{GeV}$ implies a range of $a_{2}$ of $\sim 0.42-0.05$ for the DM mass range of $0-80 \mathrm{GeV}$ thus ruling out the possibility of $\beta>0.2$ in order to prevent an overdensity of DM. Thus, the bound on $a_{2}$ from WEP constraints is a powerful probe of a dark force in the scalar singlet DM model.

For the scalar real triplet DM model, the DM relic density is determined by gauge interactions in addition to the parameter $a_{2}$. In this case, the WEP bound on $a_{2}$ shown in Fig. 8 does not necessarily rule out a dark force since the correct relic density can still be obtained from annihilation diagrams that proceed via gauge interactions that are independent of $a_{2}$. For example, the bound of $a_{2}<0.02$ implied by $\eta_{E}<10^{-13}$ for $\beta=0.2$, as shown in the top right graph of Fig. 8, implies that the annihilation rate will be dominated by gauge interactions.

\section{WEP Tests and DM-Nucleus cross sections}

The current bounds on $a_{2}$ for a nonzero $\beta$ in the dark sector, will also lead to upper bounds on the Higgsexchange contributions to the direct detection cross section. From Eq. (71), the parameter $a_{2}$ can be written in terms of the tree-level cross-section $\sigma_{\chi^{N}}$, which proceeds via a t-channel Higgs exchange, as

$$
a_{2}^{2}=\left.\left[\frac{\pi\left(M_{\chi}+m_{N}\right)^{2} m_{h}^{4}}{g_{h}^{2} v^{2} m_{N}^{2}}\right] \sigma_{\chi N}\right|_{\text {Higgs exch }} .
$$

Substituting into Eqs. (82) and (83), defining the quantities

$$
\begin{gathered}
F \equiv\left|\left(\frac{Z_{1}}{A_{1}}-\frac{Z_{2}}{A_{2}}\right)\left\{\frac{m_{e}}{m_{N}}+\sum_{q} \frac{m_{q}}{m_{N}}\left(x_{q, p}-x_{q, n}\right)\right\}\right|, \\
E \equiv\left[\frac{g_{h}\left(N_{p}+N_{n}\right)+\left(m_{e} / v\right) N_{e}}{\left(N_{p}+N_{n}\right)+\left(m_{e} / m_{N}\right) N_{e}}\right] \approx g_{h},
\end{gathered}
$$

we obtain the following relations between $\left(M_{\chi}+\right.$ $\left.m_{N}\right)^{2} \sigma_{\chi N}$ and the Higgs-exchange contributions to the DM-nucleus cross section: 


$$
\begin{aligned}
\left.\left(M_{\chi}+m_{N}\right)^{2} \sigma_{\chi N}\right|_{\text {Higgs exch }} & =\left(\frac{18}{7 \kappa}\right) g_{h}^{2}\left(\frac{m_{N}}{m_{h}}\right)^{2} \frac{1}{F} \frac{\eta_{\mathrm{DM}}}{\beta^{2}} \\
\left.\left(M_{\chi}+m_{N}\right)^{2} \sigma_{\chi N}\right|_{\text {Higgs exch }}= & \left(\frac{6 \pi}{\sqrt{7 \kappa}}\right) g_{h}^{2}\left(\frac{m_{N}}{m_{h}}\right)^{2} \\
& \times\left[\left(\frac{m_{N}}{v}\right) \frac{1}{F E}\right]^{1 / 2} \frac{\eta_{E}^{1 / 2}}{\beta} .
\end{aligned}
$$

The experimental limits on the Eötvös parameters, together with the foregoing expressions, lead to bounds on the Higgs-exchange contributions to the DM-nucleus cross sections. These bounds can be brought into the numerically
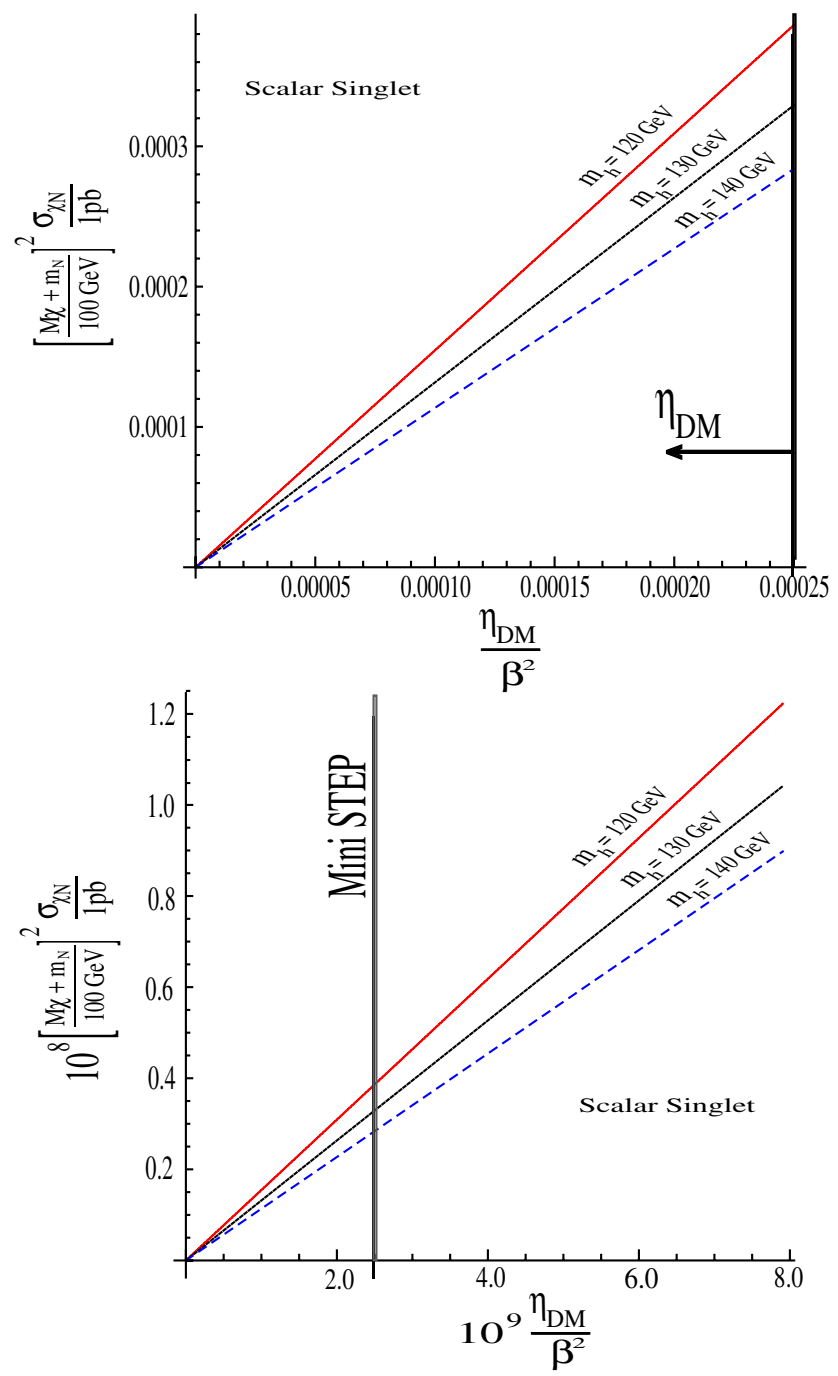

convenient form as a function of $\eta_{\mathrm{DM}} / \beta^{2}$ for the singlet $\chi$ as

$$
\begin{aligned}
{\left[\frac{M_{\chi}+m_{N}}{100 \mathrm{GeV}}\right]^{2} \frac{\sigma_{\chi N}}{1 \mathrm{pb}}<} & \left(1.1 \times 10^{4}\right) g_{h}^{2}\left[\frac{100 \mathrm{GeV}}{m_{h}}\right]^{2} \\
& \times\left|\frac{Z_{1}}{A_{1}}-\frac{Z_{2}}{A_{2}}\right|^{-1} \frac{\eta_{\mathrm{DM}}}{\beta^{2}},
\end{aligned}
$$

and for the triplet $\chi$ as

$$
\begin{aligned}
{\left[\frac{M_{\chi}+m_{N}}{2 \mathrm{TeV}}\right]^{2} \frac{\sigma_{\chi N}}{1 \mathrm{pb}}<} & 9.2 g_{h}^{2}\left[\frac{100 \mathrm{GeV}}{m_{h}}\right]^{2} \\
& \times\left|\frac{Z_{1}}{A_{1}}-\frac{Z_{2}}{A_{2}}\right|^{-1} \frac{\eta_{\mathrm{DM}}}{\beta^{2}} .
\end{aligned}
$$

Similarly, the bounds as a function of $\eta_{E} / \beta^{2}$ can be
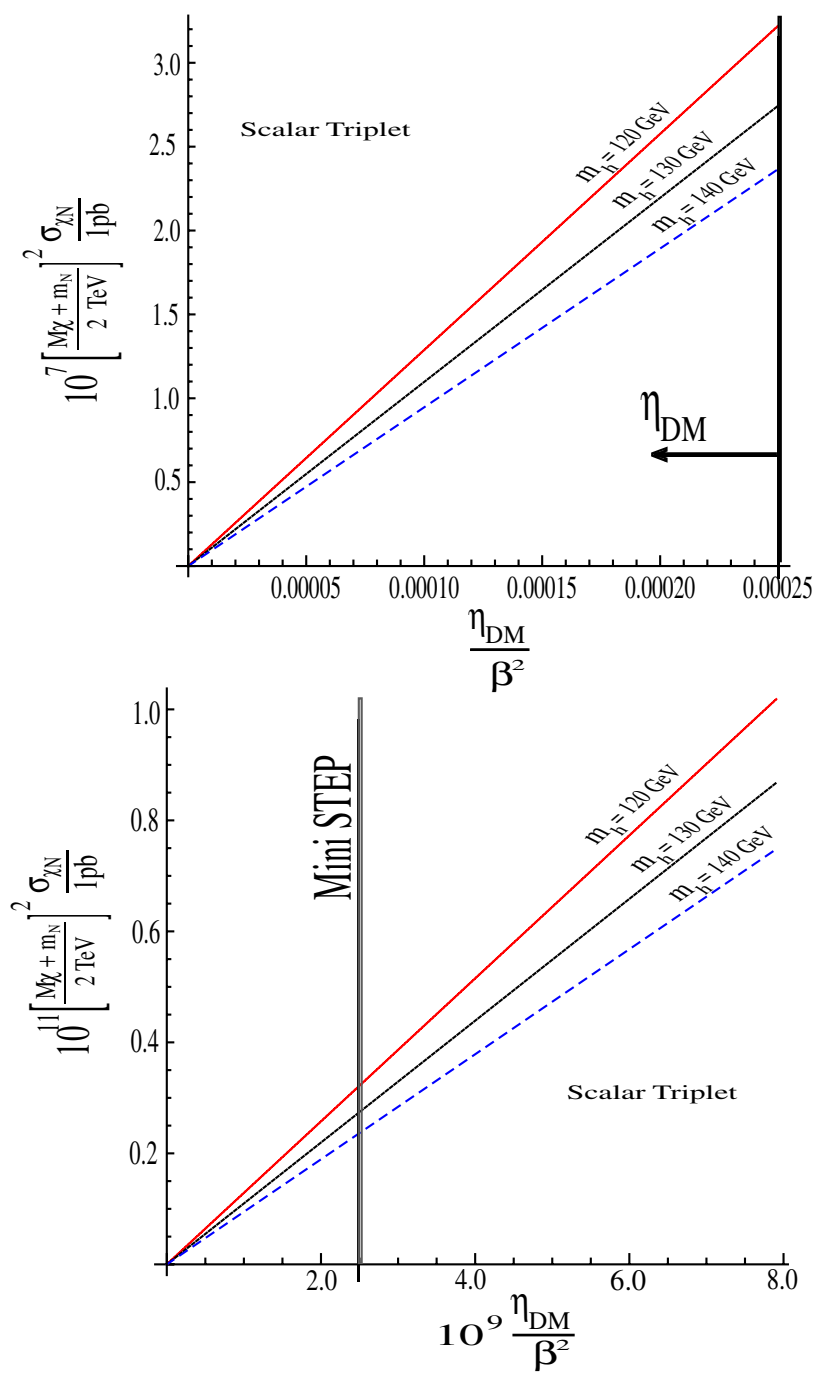

FIG. 9 (color online). Upper bounds on the Higgs-exchange tree-level direct-detection cross section of scalar singlet (left panel) and real triplet (right panel) DM implied by a dark force as a function of $\eta_{\mathrm{DM}} / \beta^{2}$. To be specific, we assume $\beta=0.2$ and discuss the implied bounds. In the top left and top right plots the vertical black line on the right corresponds to $\eta_{\mathrm{DM}}<10^{-5}$. In the bottom left and bottom right plots, the vertical black line corresponds to the expected future sensitivity of $\eta_{\mathrm{DM}}<10^{-10}$. In all plots, the three lines from to bottom correspond to the bounds for the Higgs masses of 120,130 , and $140 \mathrm{GeV}$, respectively. The size of these bounds compared to current and future sensitivities for direct-detection experiments is discussed in the text. 
brought into the numerically convenient form for the singlet $\chi$ as

$$
\begin{aligned}
{\left[\frac{M_{\chi}+m_{N}}{100 \mathrm{GeV}}\right]^{2}\left[\frac{\sigma_{\chi^{N}}}{1 \mathrm{pb}}\right]<} & 8.4 \times 10^{2} g_{h}^{2}\left[\frac{100 \mathrm{GeV}}{m_{h}}\right]^{2} \\
& \times\left|\frac{Z_{1}}{A_{1}}-\frac{Z_{2}}{A_{2}}\right|^{-1 / 2} \frac{\eta_{E}^{1 / 2}}{\beta}
\end{aligned}
$$

and for the real triplet $\chi$ as
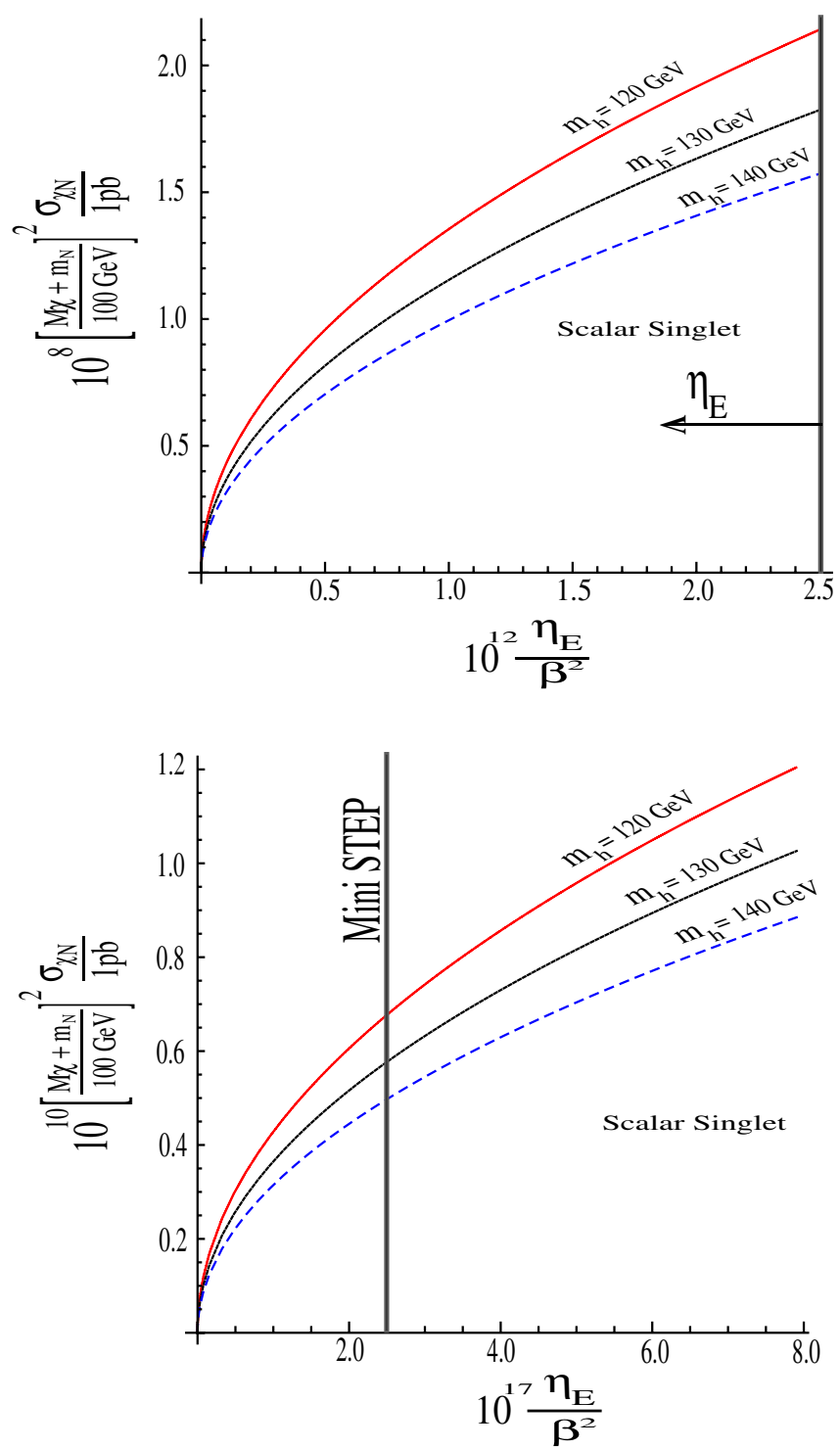

$$
\begin{aligned}
{\left[\frac{M_{\chi}+m_{N}}{2 \mathrm{TeV}}\right]^{2}\left[\frac{\sigma_{\chi N}}{1 \mathrm{pb}}\right]<} & 0.7 g_{h}^{2}\left[\frac{100 \mathrm{GeV}}{m_{h}}\right]^{2} \\
& \times\left|\frac{Z_{1}}{A_{1}}-\frac{Z_{2}}{A_{2}}\right|^{-1 / 2} \frac{\eta_{E}^{1 / 2}}{\beta},
\end{aligned}
$$

where $\left|\frac{Z_{1}}{A_{1}}-\frac{Z_{2}}{A_{2}}\right| \simeq 1 / 72$ for Beryllium and Titanium samples in Eötvös experiments. The upper bounds for the Higgs-exchange contribution to the direct-detection cross section of the singlet (left panel) and real triplet (right panel) DM in the presence of a dark force, as determined by Eqs. (89)-(92), are shown in Figs. 9 and 10 as a function
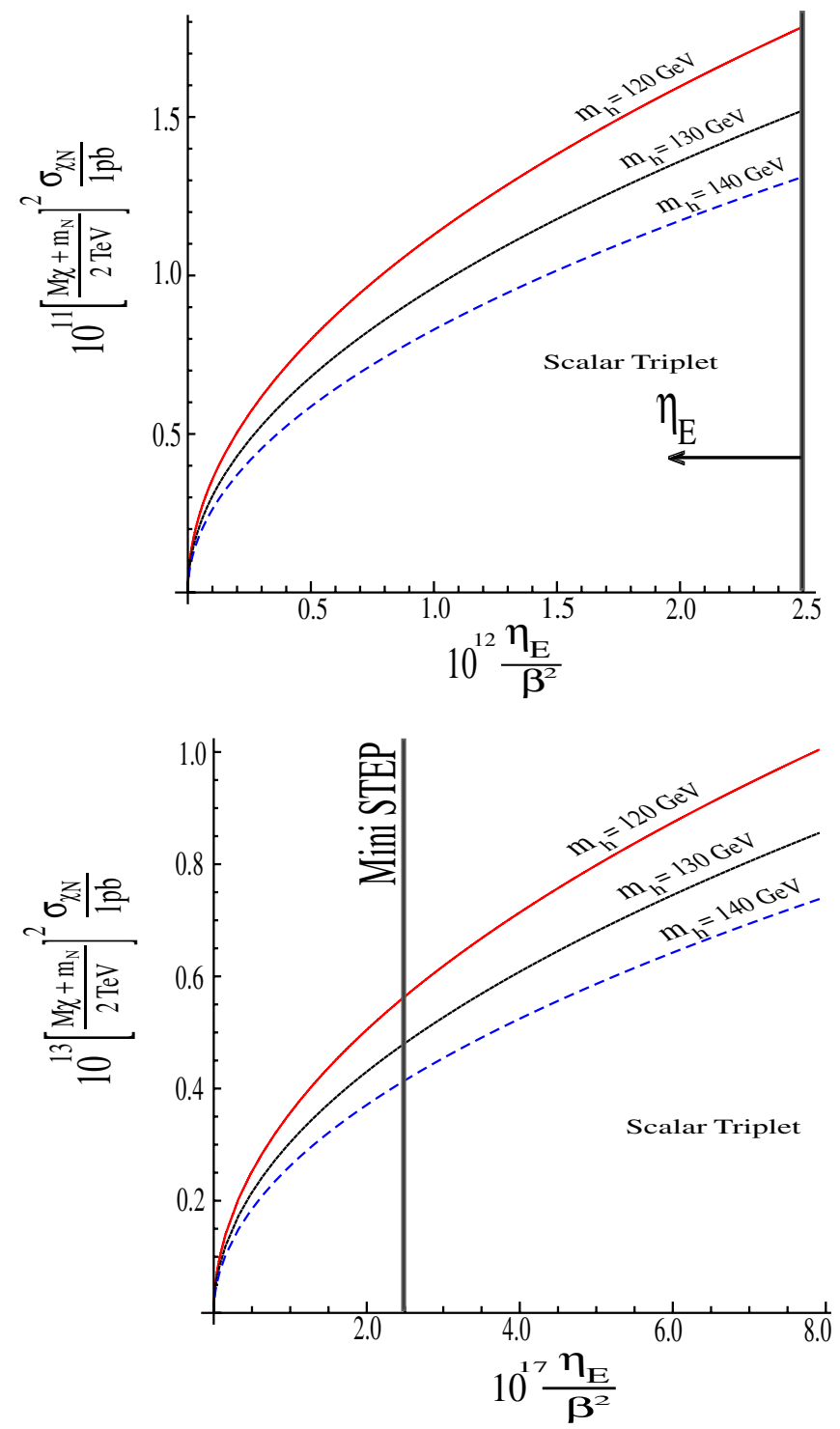

FIG. 10 (color online). Upper bounds on the Higgs-exchange tree-level direct-detection cross-section of scalar singlet (left panel) and real triplet (right panel) DM implied by a dark force as a function of $\eta_{E} / \beta^{2}$. To be specific, we assume $\beta=0.2$ and discuss the implied bounds. In the top left and top right plots, the vertical black lines on the right correspond to the current bound of $\eta_{E}<10^{-13}$. The bottom left and bottom right plots, the vertical black line corresponds to the expected future sensitivity of $\eta_{E}=10^{-18}$. In all plots, the three curves from top to bottom, correspond to the Higgs masses of 120, 130, and $140 \mathrm{GeV}$, respectively. The size of these bounds with current and future sensitivities of direct-detection experiments is discussed in the text. 
of $\eta_{\mathrm{DM}} / \beta^{2}$ and $\eta_{E} / \beta^{2}$ respectively. In each graph we show three sample curves corresponding to Higgs mass choices of $m_{h}=120,130$, and $140 \mathrm{GeV}$ as indicated. In the top row of Figs. 9 and 10, the vertical black lines on the right correspond to the current WEP bounds of $\eta_{\mathrm{DM}}<$ $10^{-5}$ and $\eta_{E}<10^{-13}$ for our benchmark value of $\beta=0.2$ which we use throughout this discussion. In the bottom row of Figs. 9 and 10, the vertical black lines labeled "MiniSTEP" correspond to the expected sensitivity of $\eta_{\mathrm{DM}} \sim 10^{-10}$ and $\eta_{E} \sim 10^{-18}$ from a possible future experiment like MiniSTEP [56]. We see that the bounds on the Higgs-exchange contribution to the DM-nucleus cross sections are typically much stronger from WEP violation constraints on $\eta_{E}$ compared to those on $\eta_{\mathrm{DM}}$. However, since the DM-nucleus cross section bounds depend linearly on $\eta_{\mathrm{DM}}$ and on the square root of $\eta_{E}$, with enough improvement the bound from $\eta_{\mathrm{DM}}$ could become stronger. In the following discussion, we focus only on the directdetection bounds from $\eta_{E}$ shown in Fig. 10.

For the scalar singlet DM, the DM-nucleus cross section bound from WEP tests does not yield any more information than the bound on $a_{2}$ which has already been discussed. This is due to the fact that $a_{2}$ determines the DM matter relic density entirely for fixed DM and Higgs masses. If the WEP violation bound on $a_{2}$ is too strong, the resulting DM relic density will be too large overclosing the universe and thus ruling out the dark force. The bound on the DM-nucleus cross section resulting from the corresponding WEP violation bound on $a_{2}$, is thus not useful since it is already ruled out.

However, the bound on the scalar singlet DM-nucleus cross section can be useful in constraining the size of $\beta$ in a multicomponent DM scenario where the scalar singlet is only a fraction of the DM. For larger values of $\beta$, as already discussed, the bounds on $a_{2}$ from WEP tests are too strong leading to an over-closed universe. For smaller values of $\beta$ the bound on $a_{2}$ becomes weaker as seen from Eqs. (82) and (83). For small enough values of $\beta$, the upper bound on $a_{2}$ would be consistent with an under-relic-density of the singlet scalar. A multicomponent DM scenario can also have $a_{2}$ consistent with an under-relic-density for the scalar singlet and in this case the WEP violation constraints on $a_{2}$ can lead to interesting bounds on the DM-nucleus cross section.

For the scalar real triplet DM, the DM relic density is determined by $a_{2}$ and gauge interactions in general. However, the tree-level DM-nucleus cross section proceeds only via a t-channel Higgs exchange and its size is determined by $a_{2}$. We point out that the bound in Fig. 10 constrains the tree-level Higgs-exchange diagram but not the one-loop diagrams, which proceed via gauge interactions and the Higgs coupling to the nucleus and is independent of $a_{2}$. Thus, if the observed DM-nucleus cross section is of the size explained by this one-loop diagram a dark force cannot be ruled out.

In Table III we show the sensitivities of current and future DM-detection experiments, taken from Table I of [57]. We see from Fig. 10 that it will be difficult for current and future direct-detection experiments to probe the upper bound on the DM-nucleus-Higgs-exchange cross sections, for scalar triplet DM, for values of $\beta$ that are astrophysically interesting allowing one to rule out this possibility. One would need a significant deviation from the expected cross section from one-loop gauge diagram, indicating a large value of $a_{2}$, to rule out a significant dark force. For smaller enough values of $\beta$, the DM-nucleus cross section bounds should be within reach of current or future experiments. The bounds we have derived on the DM-nucleus cross sections are much weaker than those in [33] since our analysis constrains higher-dimension operators with finite coefficients while the work of [33] had to rely on naturalness arguments to constrain renormalizable couplings. As we have discussed earlier, since the ultralight scalar mass is itself fine tuned we have avoided using naturalness arguments.

\section{WEP Tests and Higgs decays}

We have shown in the last section that WEP constraints lead to upper bounds on the tree-level DM-nucleus cross sections for the scalar singlet and real triplet $\chi$ models.

TABLE III. Sensitivities for DM direct detection cross sections in different experiments. These sensitivities are for $50 \mathrm{GeV}$ DM corresponding to the most sensitive mass window. We see that the XENON10, CDMS (2007), WARP (140 kg), SuperCDMS, and WARP (1 ton) experiments have enough sensitivity to probe the bounds on the direct detection cross sections in Fig. 10 for singlet DM coupled to a WEP-violating force.

\begin{tabular}{|c|c|c|c|}
\hline $\mathrm{M}_{\chi}=50 \mathrm{GeV}$ & Experiment & Sensitivity & Sensitivity \\
\hline & & $\sigma_{\chi^{N}}(\mathrm{pb})$ & {$\left[\frac{M_{X}+m_{N}}{100 \mathrm{GeV}}\right]^{2}\left[\frac{\sigma_{\chi N}}{1 \mathrm{pb}}\right]$} \\
\hline & CDMS [77] & $1.6 \times 10^{-7}$ & $4.1 \times 10^{-8}$ \\
\hline & XENON10 [17] & $4.5 \times 10^{-8}$ & $1.2 \times 10^{-8}$ \\
\hline & CDMS (2007 [78]) & $1 \times 10^{-8}$ & $3 \times 10^{-9}$ \\
\hline & WARP (140 kg) [79] & $3 \times 10^{-8}$ & $8 \times 10^{-9}$ \\
\hline & SuperCDMS (Phase A) [80] & $1 \times 10^{-9}$ & $3 \times 10^{-10}$ \\
\hline & WARP (1 ton) [81] & $2 \times 10^{-10}$ & $5 \times 10^{-10}$ \\
\hline
\end{tabular}




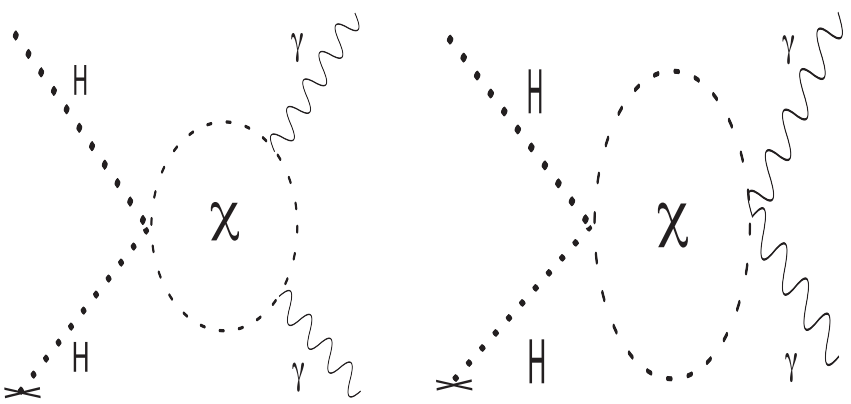

FIG. 11. Contributions to the $h \rightarrow \gamma \gamma$ rate from virtual $\chi^{ \pm}$ loops.

However, if the dark sector is made up of a rich spectrum of DM particles of different species, direct detection of any species that makes up only a tiny fraction of the relic density becomes difficult. One example of such a DM species is the neutral component of the real triplet scalar $\chi$ with a mass far below a TeV. For masses below $500 \mathrm{GeV}$, the triplet DM will make up less than $10 \%$ of the relic density [75]. The astrophysical effects of a dark force experienced by such a species would be too small to be detected. In this section, we show that when directdetection experiments or astrophysical observations fail to constrain dark forces, collider signals might still harbor information on dark forces. Fig. 2 of [76] shows the size of the shift in the $h \rightarrow \gamma \gamma$ rate for typical values of the

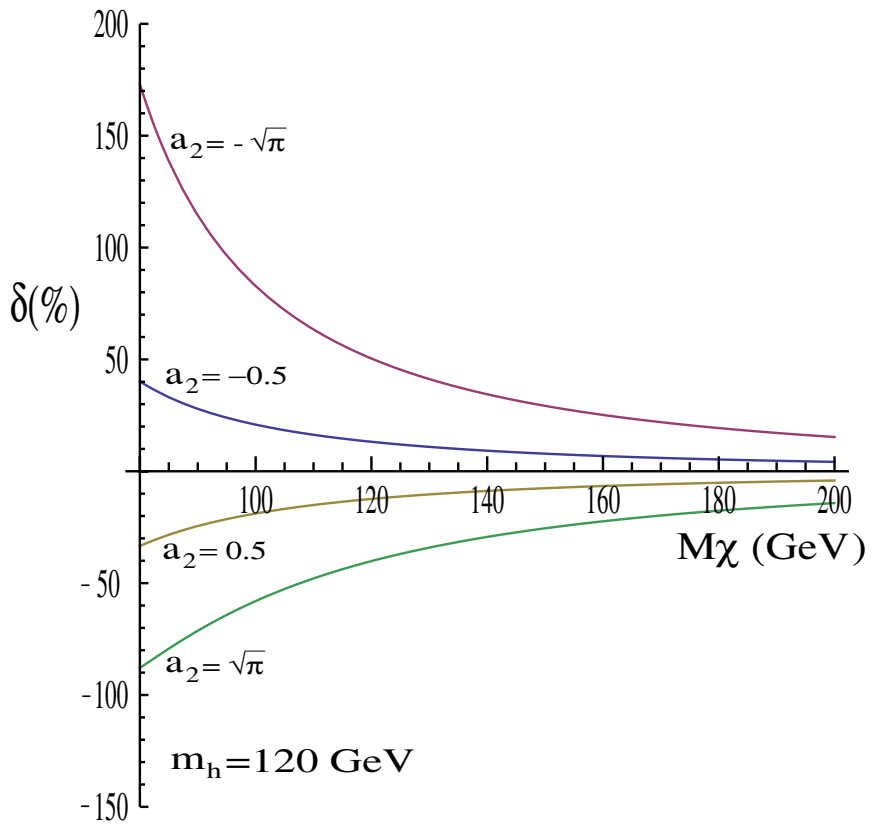

parameter $a_{2}$. We have reproduced this figure as shown on the left in Fig. 12. We plot the quantity

$$
\delta(\%) \equiv 100 \times \frac{\Gamma(h \rightarrow \gamma \gamma)-\Gamma^{\mathrm{SM}}(h \rightarrow \gamma \gamma)}{\Gamma^{\mathrm{SM}}(h \rightarrow \gamma \gamma)},
$$

For specificity we focus on the real scalar triplet $\chi$ discussed in the last section, but with a mass less than $200 \mathrm{GeV}$, and examine the implications of a dark force on collider signals. The analysis of [76] showed that one potential signature of the scalar triplet would be a modification of the $h \rightarrow \gamma \gamma$ decay rate due to the virtual charged components of the $\chi$ triplet traversing the loop shown in Fig. 11. In the rest of this section we focus on this channel. For a heavy Higgs, a similar analysis can be done for $h \rightarrow$ $\gamma Z, Z Z, W^{+} W^{-}$. As already discussed, WEP constraints imply an upper bound on the parameter $a_{2}$ which determines the size of the contribution of Fig. 11 to $h \rightarrow \gamma \gamma$. The WEP bound on $a_{2}$ translates into a bound on $\delta(\%)$ which is shown in the right panel of Fig. 12 for different values of $M_{\chi}$. Comparing the left plot of Fig. 12 with the top right graph in Fig. 8, we see that the current bounds on $a_{2}$ from $\eta_{E}$ for a nonzero $\beta$ can give nontrivial bounds on $\delta(\%)$ that can be tested in colliders. The right plot in Fig. 12 gives the upper bound on $|\delta(\%)|$ as a function of $\eta_{E} / \beta^{2}$. For $\beta=0.2$, we have the bound $\eta_{E} / \beta^{2}<2.5 \times$ $10^{12}$ coming from the current bound of $\eta_{E}<10^{-13}$. We see that the bound on $\delta(\%)$ for a dark force of $\beta=0.2$ is

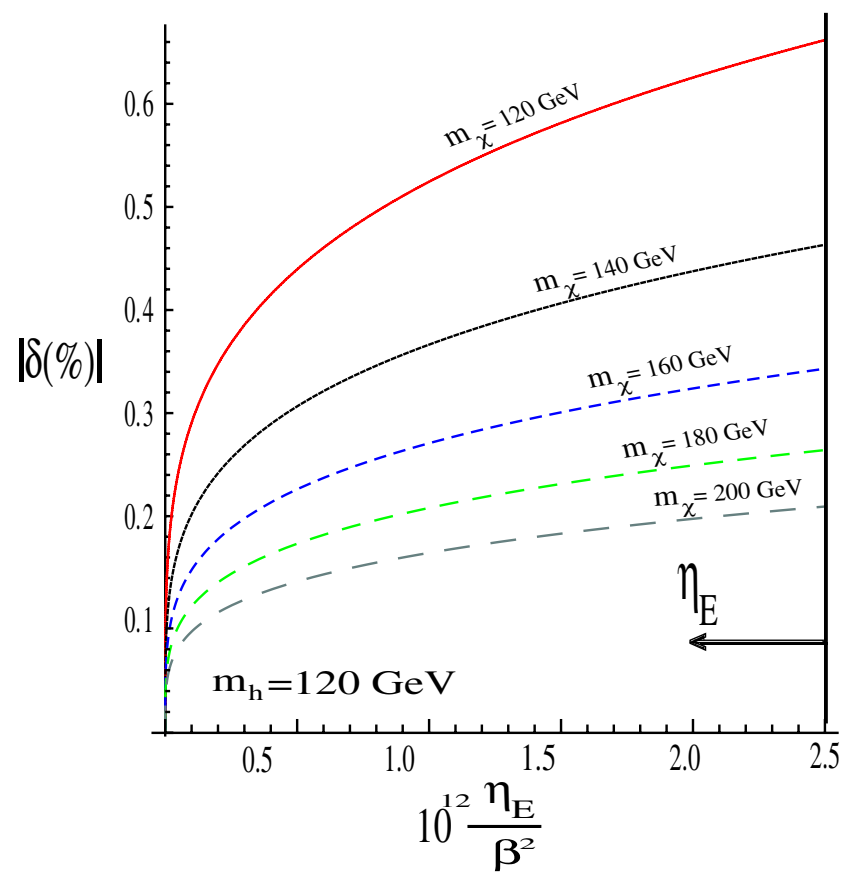

FIG. 12 (color online). The left plot shows the deviation of the $h \rightarrow \gamma \gamma$ rate compared to the SM prediction for typical values of the parameter $a_{2}$ as a function of the triplet mass $M_{\chi}$. The right plot shows the magnitude of the allowed shift in the $h \rightarrow \gamma \gamma$ rate in the presence of a dark force. The bound on this allowed shift arises due to the bound on $a_{2}$ from WEP violation constraints on $\eta_{E}$ as seen in the top right plot of Fig. 8. The typical values of $a_{2}$ in the left plot above, which lead to sizable deviations in the $h \rightarrow \gamma \gamma$ rate, are too big to be compatible with WEP violation constraints. 
well below $1 \%$. Thus, any observed shift in $h \rightarrow \gamma \gamma$, that cannot be explained by physics observed at colliders and unrelated to $\chi$, requires a significant contribution from the $\chi$ loop implying a value for $\beta$ much smaller than 0.2 . If a nonzero value of $a_{2}$ is extracted from a study of $h \rightarrow \gamma \gamma$ decays, one can estimate the size of $\eta_{\mathrm{DM}, E} / \beta^{2}$ from Eqs. (82) and (83) respectively and use the current bounds on $\eta_{\mathrm{DM}, E}$ to constrain the size of $\beta$. For example, using $m_{h}=120 \mathrm{GeV}$ and the current bound of $\eta_{E}<10^{-13}$, the nonzero values of $a_{2}=\sqrt{\pi}, 1.0,0.5$ would imply that $\beta<$ $7 \times 10^{-5}, 2 \times 10^{-4}, 9 \times 10^{-4}$ respectively.

\section{DARK FORCE PARAMETER SPACE}

Before concluding, we generally discuss the regions in parameter space of $\mathrm{SM}+\chi+\phi$ type models that are likely to give rise to an observable dark force. In particular, we discuss how the requirement $m_{\phi}<10^{-25} \mathrm{eV}$, necessary to allow a dark force of intergalactic range, restricts the allowed parameter space. Recall that after EWSB and diagonalizing the mass matrix, the ultralight scalar mass is given by

$$
m_{\phi}^{2} \simeq \mu_{S}^{2}-\frac{\mu_{h S}^{4}}{4 m_{h}^{2}} .
$$

We showed in Sec. VI A that $\mu_{h S}^{2}$, which determines the mixing between the ultralight scalar and the Higgs, receives finite contributions from higher-dimension operators whose size we constrained from WEP tests. In Eq. (80), the second term is the contribution to the mixing angle from the operator $\mathrm{H}^{\dagger} H S$ after EWSB and the first term is the finite contribution from the sum of higherdimension operators induced via DM loops (see Fig. 7). The parameter $\mu_{S}^{2}$ similarly receives finite contributions from higher-dimension operators. For example, attaching one extra external $S$ field to the DM loops in Fig. 7 will generate a tower of operators that contribute to $\mu_{S}^{2}$ after EWSB. The lowest dimension nonrenormalizable operator that contributes to $\mu_{S}^{2}$ will be

$$
D_{2} H^{\dagger} H H^{\dagger} H S^{2} \text {, }
$$

with finite coefficient $D_{2}$ which can be estimated from NDA as

$$
D_{2} \sim \frac{a_{2}^{2}}{\pi M_{P}^{2}} \beta^{2} .
$$

We could sum the contribution of the entire tower of operators to $\mu_{S}^{2}$ as we did for the case of $\mu_{h S}^{2}$. However, the explicit sum is not needed for the following discussion.

The requirement that $m_{\phi}<10^{-25} \mathrm{eV}$ now imply three types of possible regions in parameter space: I, II, and III. We discuss each of these regions in turn below and relate them to the analysis of previous sections.

Region I: In the first region of parameter space, there are no intricate cancellations of any kind among the terms in
Eq. (94). Each term that goes into determining $\mu_{S}^{2}$ and $\mu_{h S}^{2}$ is required to be of the order of $m_{\phi}^{2}$. In this case, we can obtain an approximate bound on $a_{2}$ from the $D_{2}$ which contributes to $\mu_{S}^{2} \sim m_{\phi}^{2}$ as

$$
a_{2}^{2}<\frac{4 \pi}{\beta^{2}} \frac{M_{P}^{2}}{v^{2}} \frac{m_{\phi}^{2}}{v^{2}}<\frac{3 \times 10^{-39}}{\beta^{2}} .
$$

For any observable nonzero value of $\beta$, the above bound essentially forces $a_{2}$ to be zero. As already discussed, such a small value of $a_{2}$ will lead to an over-relic-density of scalar singlet DM over-closing the universe and is ruled out. Similar arguments can be made for WIMP DM in which case $D_{2}$ will receive contributions that depend only on the gauge couplings and $\beta$, thus ruling out any observable value for $\beta$. In short, this region of parameter space is incompatible with the existence of a phenomenologically interesting dark force and thus not considered in this paper.

Region II: In this region, $\mu_{S}^{2}$ of Eq. (94) is chosen such that the condition $m_{\phi}<10^{-25} \mathrm{eV}$ is always satisfied regardless of the size of $\mu_{h S}^{2}$ and any finite contributions to it from higher-dimension operators after EWSB. Furthermore, in this region there are no intricate cancellations between terms that determine $\mu_{h S}^{2}$ so that one can put bounds on these from WEP tests. This is phenomenologically the most interesting region and was the focus of this paper.

Region III: Finally, the third region corresponds to the case where there are intricate cancellations among various terms in $\mu_{S}^{2}$ and $\mu_{h S}^{2}$ individually. If there are intricate cancellations between large terms in $\mu_{h S}^{2}$, then we cannot extract meaningful bounds on $a_{2}$ or the Higgs-exchange direct-detection cross section from WEP tests. In this special region of parameter space the bounds on $a_{2}$ and the Higgs-exchange contributions to the direct-detection cross sections derived in this paper do not apply.

The analysis of Ref. [33] assumed no fine-tuning of $\delta_{1}$ or $\mu_{h S}^{2}$ against radiative corrections sensitive to the cutoff. As seen in Eq. (94), $\mu_{h S}$ contributes to the light scalar mass $m_{\phi}$ and in the absence of fine-tuning the strongest bound on $a_{2}$ comes from $m_{\phi}<10^{-25} \mathrm{eV}$ forcing $a_{2}$ to be essentially zero. The bounds from WEP tests are relatively far weaker and not relevant for $a_{2}$ or equivalently for the DMnucleus cross section via Higgs exchange. Our analysis differs in that we allow for fine tuning in all renormalizable parameters, and then examine the different regions in the space of these renormalized parameters and the corresponding implications for terrestrial experiments.

\section{CONCLUSION}

The existence of a new long-range WEP-violating attractive force in the dark sector, a dark force comparable to gravity, can have interesting cosmological consequences, including an accelerated rate of structure formation and an 
explanation of certain features of the DM distribution and other astronomical observations [41,45,50]. Strong constraints for such a dark force comes from a study [51,52] of the dynamics of satellite galaxies and the evolution of density perturbations constrained [54] by the CMB spectrum. The current bounds indicate the strength of a possible dark force to be less than $\lesssim 20 \%$ of gravity from galactic dynamics and less than $5 \%$ of gravity from the CMB spectrum.

We considered the consequences of such a dark force for terrestrial experiments. Ordinary matter will feel the effects of a dark force via virtual DM as long as the DM candidate is not sterile. Depending on the DM model, a dark force can lead to constraints on Eötvös experiments, DM-direct-detection experiments, and Higgs decay properties to be studied at future colliders. We studied several minimal models of DM to illustrate the prospective implications of an astrophysically relevant dark force for terrestrial experiments.

(i) We derived lower bounds on the size of the Eötvös parameters $\eta_{\mathrm{DM}, E}$ for a nonzero dark force for minimal DM models. These Eötvös parameters measures the effect of new long-range forces coupling to ordinary matter which can arise through virtual DM that communicates the dark force to ordinary matter. We find that for light scalar singlet DM, relic density considerations and the experimental limits on $\eta_{E}$ rule out a dark force having strength of $20 \%$ of gravity, in large regions of parameter space. Future experiments with improved sensitivity could probe a dark force of this magnitude for heavier singlet DM. For minimal WIMP DM, the expected magnitudes of $\eta_{\text {DM,E }}$ lie well below current and prospective sensitivities of terrestrial Eötvös experiments, but could be probed in a satellite-based experiment having the sensitivity of the MiniSTEP proposal. In nonminimal WIMP DM models, it is possible to generate larger effects for Eötvös experiments that could be detected by the Microscope experiment.

(ii) WEP tests imply constraints on Higgs-exchange contributions to the DM-nucleus cross sections. For scalar singlet DM, these bounds apply to the entire cross section. If the scalar singlet DM saturates the DM relic density, these bounds on the DMnucleus cross section do not give any information beyond the implications of WEP bounds on the DM relic density. If the scalar singlet does not saturate the DM relic density, allowing for stronger interactions with the Higgs, WEP tests provide useful bounds on the DM-nucleus cross section. For WIMP DM, the WEP constraints on the HiggsDM interactions give upper bounds on the contribution of DM-nucleus scattering via Higgs exchange, to the total cross section.

(iii) For singlet DM, the current WEP bounds on the DM-nucleus cross section in the presence of a dark force that is $20 \%$ of gravity, are typically within reach of current and future direct-detection experiments. For scalar WIMP DM with a mass in the $\mathrm{TeV}$ range, the corresponding bounds on the DMnucleus cross section are typically beyond the reach of current and future direct-detection experiments. If these scalar WIMPs are detected, it will rule out a dark force greater than $20 \%$ of gravity, implying a tighter upper bound on the dark force.

(iv) The Eötvös constraints on the DM-Higgs interactions can lead to constraints for collider physics. As a specific example, we derived testable bounds on the allowed shift in the $h \rightarrow \gamma \gamma$ rate when the Higgs couples to WIMP DM in the real triplet scalar representation with a mass less than $200 \mathrm{GeV}$. Such a light triplet will only contribute a tiny fraction of the DM relic density and could be part of a multicomponent DM scenario. The implied bounds on the Higgs to two photon rate or a dark force comparable to gravity, is far below the sensitivity of the LHC or the ILC. An observed shift in $h \rightarrow \gamma \gamma$ attributed to the charged components of the triplet would rule out the dark force.

(v) An observable scalar dark force with intergalactic range implies restrictions in the space of the renormalized parameters of the theory. These parameter space restrictions apply after the usual fine-tuning of parameters against radiative corrections sensitive to the cutoff.

Apart from these experimental implications, a notable theoretical consequence of an astrophysically interesting dark force mediated by an ultralight scalar $\phi$ is the need for substantial fine-tuning to preserve its tiny mass $\left(m_{\phi}<\right.$ $10^{-25} \mathrm{eV}$ ). In the DM scenarios considered here, divergent loop contributions associated with the DM or SM particles that interact with $\phi$ would generate large contributions to $m_{\phi}$ that must be removed by fine-tuning unless the strength of the dark force is imperceptibly small. The discovery of such a dark force would introduce yet another mass hierarchy problem in particle physics. In our analysis, we have taken this need for fine-tuning at face value and have attempted to apply it consistently to the derivation of implications for terrestrial experiments. These consequences imply that direct-detection experiments-together with Eötvös experiments and astrophysical observations of satellite galaxies and structure formation-can be employed as part of a multifaceted probe of a long-range force in the dark sector.

\section{ACKNOWLEDGMENTS}

We would like to thank Daniel Chung, Glennys Farrar, Jens Gundlach, Marc Kamionkowski, Thomas McElmurry, Frank Petriello, and Lian-Tao Wang for useful discussions. This work was supported in part by Department of Energy contracts DE-FG03-92-ER40701 
and DE-FG02-08ER41531, the Wisconsin Alumni Research Foundation, the Alfred P. Sloan Foundation, and the Gordon and Betty Moore Foundation.

\section{APPENDIX A: ATOMIC CHARGE-TO-MASS RATIO UNDER A COUPLING TO ULTRALIGHT SCALARS}

Here we give details for obtaining the atomic charge-tomass ratio for a coupling to ultralight scalars following [66]. As discussed in the text and shown in Eq. (20), we can obtain the atomic coupling to $\phi$ from the couplings of $\phi$ to SM particles by a matching calculation. From Eqs. (19) and (20), the coupling atomic coupling $g_{A}$ is given by

$$
\begin{aligned}
\xi_{A} g_{A}= & \frac{1}{m_{p}}\left\langle A\left|\left(\sum_{q} g_{q} m_{q} \bar{q} q+\sum_{\ell} g_{\ell} m_{\ell} \bar{\ell} \ell\right)\right| A\right\rangle \\
& +c_{g}\left\langle A\left|G_{a}^{\mu \nu} G_{\mu \nu}^{a}\right| A\right\rangle+c_{\gamma}\left\langle A\left|F^{\mu \nu} F_{\mu \nu}\right| A\right\rangle .
\end{aligned}
$$

Note that with the normalization factor $\xi_{A}$ the above equation is dimensionally consistent, since for fermionic atoms $g_{A}$ is dimensionless and for scalar atoms it has dimension one. Next we exploit the properties of the energy-momentum tensor in the low-energy effective theory. The trace of the energy-momentum tensor, after using on-shell equations of motion, $[67,82]$ is given by

$$
\begin{aligned}
\theta_{\mu}^{\mu}= & \frac{\beta_{3}}{2 g_{3}} G_{\mu \nu}^{a} G_{a}^{\mu \nu}+\frac{\beta_{e}}{2 e} F_{\mu \nu} F^{\mu \nu}+\sum_{q}\left(1+\gamma_{m_{q}}\right) m_{q} \bar{q} q \\
& +\sum_{\ell}\left(1+\gamma_{m_{\ell}}\right) m_{\ell} \bar{\ell} \ell
\end{aligned}
$$

where in this convention the QCD and QED beta functions at one loop are

$$
\begin{aligned}
& \beta_{3}=-\left[\frac{11}{3} C_{2}(G)-\frac{n_{f}}{2} C_{2}\left(N_{c}\right)\right] \frac{g_{3}^{3}}{16 \pi^{2}}, \\
& \beta_{e}=\frac{e^{3}}{12 \pi^{2}} n_{f},
\end{aligned}
$$

where $C_{2}(G)=4 / 3$ and $C_{2}\left(N_{c}\right)=3$ for $N_{c}=3$. In the following we ignore the anomalous dimensions $\gamma_{m_{q}, m_{\ell}}$ in the factors $\left(1+\gamma_{m_{q}, m_{\ell}}\right)$ since they are perturbatively suppressed. The mass of the atom $A$ is given by

$$
\begin{aligned}
M_{A}= & \left\langle A\left|\theta_{\mu}^{\mu}\right| A\right\rangle \\
= & \left\langle A\left|\left(\frac{\beta_{3}}{2 g_{3}} \operatorname{Tr}\left[G_{\mu \nu} G^{\mu \nu}\right]+\frac{\beta_{e}}{2 e} F_{\mu \nu} F^{\mu \nu}\right)\right| A\right\rangle \\
& +\left\langle A\left|\left(\sum_{q} m_{q} \bar{q} q+\sum_{\ell} m_{\ell} \bar{\ell} \ell\right)\right| A\right\rangle .
\end{aligned}
$$

The atomic mass can also be expressed as

$$
M_{A}=Z m_{p}+(A-Z) m_{n}+Z m_{e}-\mathcal{E}_{A},
$$

where $\mathcal{E}_{A}$ is the binding energy of the atom $A$. From here one can write the derivative with respect to the quark and lepton masses as

$m_{q} \frac{d M_{A}}{d m_{q}}=Z m_{q} \frac{d m_{p}}{d m_{q}}+(A-Z) m_{q} \frac{d m_{n}}{d m_{q}}-m_{q} \frac{d \mathcal{E}}{d m_{q}}$,

$m_{\ell} \frac{d M_{A}}{d m_{\ell}}=Z m_{e} \delta_{e \ell}-m_{\ell} \frac{d \mathcal{E}}{d m_{\ell}}$.

We now use Eq. (A4) for $M_{A}$ and the Feynman-Hellman theorem to calculate $m_{q, \ell} \frac{d M_{A}}{d m_{q, \ell}}$ and equate with Eq. (A6), we obtain

$$
\begin{aligned}
m_{q} \frac{d M_{A}}{d m_{q}} & =\left\langle A\left|m_{q} \bar{q} q\right| A\right\rangle \\
& =Z m_{q} \frac{d m_{p}}{d m_{q}}+(A-Z) m_{q} \frac{d m_{n}}{d m_{q}}-m_{q} \frac{d \mathcal{E}_{A}}{d m_{q}}, \\
m_{\ell} \frac{d M_{A}}{d m_{\ell}} & =\left\langle A\left|m_{\ell} \bar{\ell} \ell\right| A\right\rangle=Z m_{e} \delta_{e \ell}-m_{\ell} \frac{d \mathcal{E}_{A}}{d m_{\ell}} .
\end{aligned}
$$

Using the relations of Eq. (A7) in Eq. (A4) we obtain an expression for the atomic matrix element of the gluon operator as

$$
\begin{aligned}
\langle A| & \frac{\beta_{3}}{2 g_{3}} \operatorname{Tr}\left[G_{\mu \nu} G^{\mu \nu}\right]|A\rangle \\
= & M_{A}-Z\left(m_{e}+\sum_{q} m_{q} \frac{d m_{p}}{d m_{q}}\right)-(A-Z) \sum_{q} m_{q} \frac{d m_{n}}{d m_{q}} \\
& +\sum_{k} m_{k} \frac{d \mathcal{E}_{A}}{d m_{k}}-\left\langle A\left|\frac{\beta_{e}}{2 e} F_{\mu \nu} F^{\mu \nu}\right| A\right\rangle,
\end{aligned}
$$

where the sum over $k$ runs over the quark and lepton masses. Using Eqs. (A7) and (A8) in Eq. (A1) we finally arrive at the expression for $\xi_{A} g_{A}$ :

$$
\begin{aligned}
\xi_{A} g_{A}= & \frac{2 c_{g} g_{3}}{\beta_{3}} M_{A}+\left[Z\left(\zeta_{e} m_{e}+\sum_{q} \zeta_{q} m_{q} \frac{d m_{p}}{d m_{q}}\right)\right. \\
& \left.+(A-Z) \sum_{q} \zeta_{q} m_{q} \frac{d m_{n}}{d m_{q}}-\sum_{k} \zeta_{k} m_{k} \frac{d \mathcal{E}_{A}}{d m_{k}}\right] \\
& +\kappa\left\langle A\left|F^{\mu \nu} F_{\mu \nu}\right| A\right\rangle,
\end{aligned}
$$

where we have introduced the index $k$ which runs over the light quarks $q$ and the charged leptons $\ell$ and the parameters $\zeta_{k}$ and $\kappa$ are given by

$$
\zeta_{k}=\frac{g_{k}}{m_{p}}-\frac{2 g_{3}}{\beta_{3}} c_{g}, \quad \kappa=c_{\gamma}-\frac{g_{3} \beta_{e}}{e \beta_{3}} c_{g} .
$$

We now utilize the expression for the nucleon mass in terms of the nucleon matrix element of the trace of the three flavor QCD energy-momentum tensor

$$
m_{N}=\left\langle N\left|\theta_{\mu}^{\mu}\right| N\right\rangle=\left\langle N\left|\frac{\beta_{3}}{2 g_{3}} G_{\mu \nu}^{a} G_{a}^{\mu \nu}+\sum_{q} m_{q} \bar{q} q\right| N\right\rangle,
$$

the variation of the nucleon mass with respect to the mass 
of a quark of flavor $q$ is given by

$$
m_{q} \frac{d m_{N}}{d m_{q}}=\left\langle N\left|m_{q} \bar{q} q\right| N\right\rangle .
$$

Once again we have used the nonrelativistic normalization of nucleon states. The nucleon matrix elements on the rhs are extracted from pion-nucleon scattering data using chiral perturbation theory. Experimentally their values are determined to be $[67,70]$

$$
\begin{aligned}
& x_{u, p} \equiv \frac{d m_{p}}{d m_{u}}=\langle p|\bar{u} u| p\rangle \sim 0.019 m_{p} / m_{u}, \\
& x_{d, p} \equiv \frac{d m_{p}}{d m_{d}}=\langle p|\bar{d} d| p\rangle \sim 0.041 m_{p} / m_{d}, \\
& x_{s, p} \equiv \frac{d m_{p}}{d m_{s}}=\langle p|\bar{s} s| p\rangle \sim 0.14 m_{p} / m_{s}, \\
& x_{u, n} \equiv \frac{d m_{n}}{d m_{u}}=\langle n|\bar{u} u| n\rangle \sim 0.023 m_{n} / m_{u}, \\
& x_{d, n} \equiv \frac{d m_{n}}{d m_{d}}=\langle n|\bar{d} d| n\rangle \sim 0.034 m_{n} / m_{d}, \\
& x_{s, n} \equiv \frac{d m_{n}}{d m_{s}}=\langle n|\bar{s} s| n\rangle \sim 0.14 m_{n} / m_{s} .
\end{aligned}
$$

These numbers are taken from Table 6 of [67]. In general $m_{k} d \mathcal{E}_{A} / d m_{k}$ and $\left\langle A\left|F^{\mu \nu} F_{\mu \nu}\right| A\right\rangle$ are not analytically calculable, at least for large atoms, and will contribute to the uncertainty in the atomic charge-to-mass ratio. The atomic charge-to-mass ratio can be finally written as

$$
\begin{aligned}
\hat{\xi}_{A}\left(\frac{q}{\mu}\right)_{A}= & \frac{g_{A} \xi_{A}}{M_{A}} \\
= & \frac{2 c_{g} g_{3}}{\beta_{3}}+\frac{1}{M_{A}}\left[Z\left(\zeta_{e} m_{e}+\sum_{q} \zeta_{q} m_{q} x_{q, p}\right)\right. \\
& \left.+(A-Z) \sum_{q} \zeta_{q} m_{q} x_{q, n}+\omega_{A}\right]
\end{aligned}
$$

where we have defined

$$
\omega_{A} \equiv \kappa\left\langle A\left|F^{\mu \nu} F_{\mu \nu}\right| A\right\rangle-\sum_{k} \zeta_{k} m_{k} \frac{d \mathcal{E}_{A}}{d m_{k}} .
$$

\section{APPENDIX B: EFFECTIVE POTENTIAL FOR HIGGS-ULTRALIGHT-SCALAR MIXING}

As discussed in Sec. VI, ordinary matter can couple to the ultralight scalar $\phi$, which mediates a long-range WEPviolating force, via its mixing with the Higgs. Here we show the computation of the effective potential which generates this mixing after electroweak symmetry breaking. This effective potential is generated at one loop via the sum of diagrams shown in Fig. 7 for the scalar singlet $\chi$ and real scalar triplet $\chi$ models discussed in Sec. VI. Working in unitary gauge where $H=h / \sqrt{2}$ and in d- dimensions, one can write the sum of all diagrams in Fig. 7 as

$$
\begin{aligned}
-i V_{\mathrm{eff}}^{S}(S, h)= & -i \kappa g_{\chi} S \int_{E} \frac{d^{d} k}{(2 \pi)^{d}} \sum_{n=0}^{\infty} \frac{\left(a_{2} h^{2}\right)^{n}}{\left(k^{2}+M_{0}^{2}\right)^{n+1}} \\
= & -i \kappa g_{\chi} S \int_{E} \frac{d^{d} k}{(2 \pi)^{d}} \frac{1}{\left(k^{2}+M_{0}^{2}+a_{2} h^{2}\right)} \\
= & \kappa \frac{i g_{\chi} S}{16 \pi^{2}}\left(M_{0}^{2}+a_{2} h^{2}\right)\left[\frac{1}{\epsilon}-\gamma_{E}+\ln 4 \pi+1\right. \\
& \left.-\ln \left(\frac{M_{0}^{2}+a_{2} h^{2}}{\mu^{2}}\right)\right],
\end{aligned}
$$

where the first line is obtained after performing a Wick rotation to Euclidean momentum space. The superscript in $V_{\text {eff }}^{S}(S, h)$ denotes that it is only the part of the effective potential linear in $S$. We see from the above result that the coefficient of the $S$ and $S h^{2}$ operators are UV divergent. These divergences are understood from the need to renormalize the tadpole graph of $S$ and the renormalizable coupling $\delta_{1}$ of Eq. (38), corresponding to the first two diagrams in Fig. 7. The remaining diagrams mix into nonrenormalizable operators and are finite. The counterterms needed to cancel the UV divergences are

$$
\begin{aligned}
i \delta V_{\mathrm{eff}}(S, h)= & S\left[\kappa \frac{g_{\chi} M_{0}^{2}}{16 \pi^{2}}\left(\frac{1}{\epsilon}-\gamma_{E}+\ln 4 \pi\right)+\hat{b}_{1}(\mu)\right] \\
& +S h^{2}\left[\kappa \frac{g_{\chi} a_{2}}{16 \pi^{2}}\left(\frac{1}{\epsilon}-\gamma_{E}+\ln 4 \pi\right)+\frac{\hat{\delta}_{1}(\mu)}{4}\right],
\end{aligned}
$$

where $\hat{b}_{1}(\mu)$ and $\hat{\delta}_{1}(\mu)$ are scheme dependent finite quantities.

The quadratic terms in the potential is given by

$$
V_{\text {quad }}=\frac{1}{2}\left(\mu_{h}^{2} h^{2}+\mu_{S}^{2} S^{2}+\mu_{h S}^{2} h S\right),
$$

as first shown in Eq. (40). As seen from Eqs. (76) and (80) the mixing angle for Higgs-ultralight-scalar mixing is given by

$$
\sin \theta \simeq \frac{\mu_{h S}^{2}}{\mu_{h}^{2}} \simeq \frac{\mu_{h S}^{2}}{m_{h}^{2}}
$$

and we can write

$$
\begin{aligned}
\mu_{h S}^{2} & =2 \frac{\partial^{2} V_{\mathrm{quad}}}{\partial S \partial h}=\left.2 \frac{\partial^{2} V_{\mathrm{eff}}(h, S)}{\partial S \partial h}\right|_{h=v, S=0}, \\
& =v\left[\hat{\delta}_{1}(\mu)+\kappa \frac{g_{\chi} a_{2}}{4 \pi^{2}}\left(\ln \frac{M_{\chi}^{2}}{\mu^{2}}-1\right)\right]+\kappa \frac{a_{2}^{2}}{4 \pi} \frac{g_{\chi} v^{3}}{M_{\chi}^{2}},
\end{aligned}
$$

where we have defined the renormalized effective potential $\mathcal{V}_{\text {eff }}$ as 


$$
\mathcal{V}_{\text {eff }} \equiv V_{\text {eff }}+\delta V_{\text {eff }}
$$

The first term with square brackets in Eq. (B5) corresponds to the renormalized value of $v \delta_{1}$ and the last term corresponds to the finite contribution from all nonrenormalizable operators. This can be compared to Eq. (76) where we have included only the contribution from the renormalized $\delta_{1}$ coupling and the third diagram in Fig. 7 whose Wilson coefficient is denoted as $C_{2}$. The above result, which is given in Eq. (79) of the text, is the generalized result where the contribution of the entire tower of higher-dimension operators is resummed.
[1] , S. Perlmutter et al. (Supernova Cosmology Project), Astrophys. J. 517, 565 (1999).

[2] A. G. Riess et al. (Supernova Search Team), Astron. J. 116, 1009 (1998).

[3] , B. P. Schmidt et al. (Supernova Search Team), Astrophys. J. 507, 46 (1998).

[4] P.M. Garnavich et al. (Supernova Search Team), Astrophys. J. 509, 74 (1998).

[5] R. A. Knop et al. (Supernova Cosmology Project), Astrophys. J. 598, 102 (2003).

[6] S. M. Faber and J.S. Gallagher, Annu. Rev. Astron. Astrophys. 17, 135 (1979).

[7] A. Bosma, Astron. J. 86, 1825 (1981).

[8] V. C. Rubin, D. Burstein, W. K.. Ford, and N. Thonnard, Astrophys. J. 289, 81 (1985).

[9] W. $\mathrm{Hu}$ and N. Sugiyama, Astrophys. J. 444, 489 (1995).

[10] W. Hu and N. Sugiyama, Phys. Rev. D 51, 2599 (1995).

[11] G. Jungman, M. Kamionkowski, A. Kosowsky, and D. N. Spergel, Phys. Rev. D 54, 1332 (1996).

[12] M. Zaldarriaga, D. N. Spergel, and U. Seljak, Astrophys. J. 488, 1 (1997).

[13] D. J. Eisenstein and W. Hu, Astrophys. J. 496, 605 (1998).

[14] D. J. Eisenstein et al. (SDSS), Astrophys. J. 633, 560 (2005).

[15] D. Clowe et al., Astrophys. J. 648, L109 (2006).

[16] P. Zhang, M. Liguori, R. Bean, and S. Dodelson, Phys. Rev. Lett. 99, 141302 (2007).

[17] J. Angle et al. (XENON), Phys. Rev. Lett. 100, 021303 (2008).

[18] Z. Ahmed et al. (CDMS Collaboration), Phys. Rev. Lett. 102, 011301 (2009).

[19] O. Adriani et al., Nature (London) 458, 607 (2009).

[20] S. W. Barwick et al. (HEAT), Astrophys. J. 482, L191 (1997).

[21] J. J. Beatty et al., Phys. Rev. Lett. 93, 241102 (2004).

[22] M. Aguilar et al. (AMS-01), Phys. Lett. B 646, 145 (2007).

[23] S. Gardner, Phys. Rev. Lett. 100, 041303 (2008).

[24] S. Gardner, Phys. Rev. D 79, 055007 (2009).

[25] A. De Rujula, S. L. Glashow, and U. Sarid, Nucl. Phys. B333, 173 (1990).

[26] J. L. Feng, H. Tu, and H.-B. Yu, J. Cosmol. Astropart. Phys. 10 (2008) 043.

[27] N. Arkani-Hamed, D. P. Finkbeiner, T. Slatyer, and N. Weiner, Phys. Rev. D 79, 015014 (2009).

[28] L. Ackerman, M. R. Buckley, S.M. Carroll, and M. Kamionkowski, Phys. Rev. D 79, 023519 (2009).
[29] M. Baumgart, C. Cheung, J. T. Ruderman, L.-T. Wang, and I. Yavin, J. High Energy Phys. 04 (2009) 014.

[30] A. Aguirre, C. P. Burgess, A. Friedland, and D. Nolte, Classical Quantum Gravity 18, R223 (2001).

[31] K. M. Zurek, Phys. Rev. D 79, 115002 (2009).

[32] Y. Bai and Z. Han, Phys. Rev. Lett. 103, 051801 (2009).

[33] J. Bovy and G. R. Farrar, arXiv:0807.3060.

[34] S. M. Carroll, S. Mantry, M. J. Ramsey-Musolf, and C. W. Stubbs, Phys. Rev. Lett. 103, 011301 (2009).

[35] T. Damour, G. W. Gibbons, and C. Gundlach, Phys. Rev. Lett. 64, 123 (1990).

[36] J. A. Frieman and B.-A. Gradwohl, Phys. Rev. Lett. 67, 2926 (1991).

[37] B.-A. Gradwohl and J. A. Frieman, Astrophys. J. 398, 407 (1992).

[38] G.W. Anderson and S.M. Carroll, arXiv:astro-ph/ 9711288.

[39] S. M. Carroll, Phys. Rev. Lett. 81, 3067 (1998).

[40] L. Amendola and D. Tocchini-Valentini, Phys. Rev. D 66, 043528 (2002).

[41] G. R. Farrar and P. J.E. Peebles, Astrophys. J. 604, 1 (2004).

[42] S. S. Gubser and P. J. E. Peebles, Phys. Rev. D 70, 123511 (2004).

[43] S. S. Gubser and P. J. E. Peebles, Phys. Rev. D 70, 123510 (2004).

[44] O. Bertolami and J. Paramos, Phys. Rev. D 71, 023521 (2005).

[45] A. Nusser, S. S. Gubser, and P. J. E. Peebles, Phys. Rev. D 71, 083505 (2005).

[46] R. Bean, E. E. Flanagan, and M. Trodden, Phys. Rev. D 78, 023009 (2008).

[47] J. M. Alimi and A. Fuzfa, J. Cosmol. Astropart. Phys. 09 (2008) 014.

[48] A. Fuzfa and J. M. Alimi, Phys. Rev. D 75, 123007 (2007).

[49] A. Coc, K. A. Olive, J.-P. Uzan, and E. Vangioni, Phys. Rev. D 79, 103512 (2009).

[50] J. Einasto et al. (SDSS), Astron. Astrophys. 462, 397 (2007).

[51] M. Kesden and M. Kamionkowski, Phys. Rev. D 74, 083007 (2006).

[52] M. Kesden and M. Kamionkowski, Phys. Rev. Lett. 97, 131303 (2006).

[53] M.-Y. Chou et al., Astrophys. J. 670, 346 (2007.

[54] R. Bean, E. E. Flanagan, I. Laszlo, and M. Trodden, Phys. Rev. D 78, 123514 (2008).

[55] C. Lammerzahl, C. W. F. Everitt, and F. W. Hehl, Prepared for 220th WE-Heraeus Seminar on Gyros, Clocks, and 
Interferometers: Testing General Relativity in Space, Bad Honnef, Germany, 1999, Berlin, (Springer 2001).

[56] N. A. Lockerbie, Nucl. Phys. B, Proc. Suppl. 61B, 3 (1998).

[57] V. Barger, P. Langacker, M. McCaskey, M. J. RamseyMusolf, and G. Shaughnessy, Phys. Rev. D 77, 035005 (2008).

[58] X.-G. He, T. Li, X.-Q. Li, J. Tandean, and H.-C. Tsai, Phys. Rev. D 79, 023521 (2009).

[59] V. Barger, P. Langacker, M. McCaskey, M. RamseyMusolf, and G. Shaughnessy, Phys. Rev. D 79, 015018 (2009).

[60] S. Schlamminger, K. Y. Choi, T. A. Wagner, J.H. Gundlach, and E. G. Adelberger, Phys. Rev. Lett. 100, 041101 (2008).

[61] J. G. Williams, S. G. Turyshev, and T. W. Murphy, and W. Thomas, Int. J. Mod. Phys. D 13, 567 (2004).

[62] S. Dimopoulos, P. W. Graham, J. M. Hogan, and M. A. Kasevich, Phys. Rev. Lett. 98, 111102 (2007).

[63] J. A. Keselman, A. Nusser, and P. J. E. Peebles, Phys. Rev. D 80, 063517 (2009).

[64] M. A. Shifman, A. I. Vainshtein, and V. I. Zakharov, Phys. Lett. B 78, 443 (1978).

[65] M. Drees and M. Nojiri, Phys. Rev. D 48, 3483 (1993).

[66] D. B. Kaplan and M. B. Wise, J. High Energy Phys. 08 (2000) 037.

[67] G. Jungman, M. Kamionkowski, and K. Griest, Phys. Rep.
267, 195 (1996).

[68] J.E. Moody and F. Wilczek, Phys. Rev. D 30, 130 (1984).

[69] C. D. Hoyle et al., Phys. Rev. D 70, 042004 (2004).

[70] G. Belanger, F. Boudjema, A. Pukhov, and A. Semenov, Comput. Phys. Commun. 180, 747 (2009).

[71] T. P. Cheng, Phys. Rev. D 38, 2869 (1988).

[72] H.-Y. Cheng, Phys. Lett. B 219, 347 (1989).

[73] C. P. Burgess, M. Pospelov, and T. ter Veldhuis, Nucl. Phys. B619, 709 (2001).

[74] D. O'Connell, M. J. Ramsey-Musolf, and M. B. Wise, Phys. Rev. D 75, 037701 (2007).

[75] M. Cirelli, N. Fornengo, and A. Strumia, Nucl. Phys. B753, 178 (2006).

[76] P. Fileviez Perez, H. H. Patel, M. J. Ramsey-Musolf, and K. Wang, Phys. Rev. D 79, 055024 (2009).

[77] D. S. Akerib et al. (CDMS Collaboration), Phys. Rev. D 73, 011102 (2006)

[78] K. Ni and L. Baudis, Adv. Space Res. 41, 2019 (2008).

[79] R. Brunetti et al., (WARP)New Astron. Rev. 49, 265 (2005).

[80] D. S. Akerib et al., Nucl. Instrum. Methods Phys. Res., Sect. A 559, 411 (2006).

[81] E. Aprile et al., arXiv:astro-ph/0207670.

[82] J. C. Collins, A. Duncan, and S. D. Joglekar, Phys. Rev. D 16, 438 (1977). 